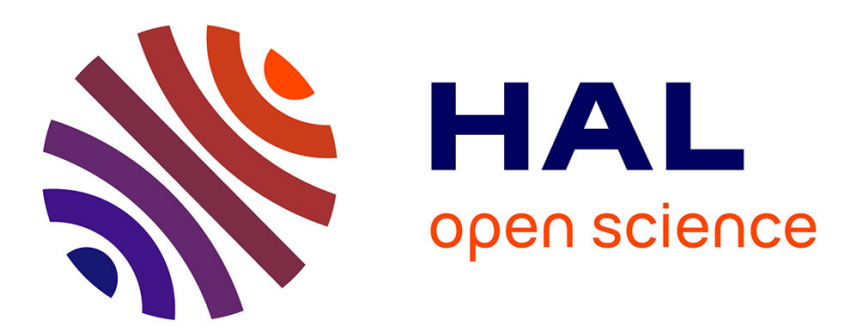

\title{
Effect of rotation on the stability of side-heated buoyant convection between infinite horizontal walls
}

Abdessamed Medelfef, Daniel Henry, Ahcene Bouabdallah, Slim Kaddeche, Redouane Boussaa

\section{- To cite this version:}

Abdessamed Medelfef, Daniel Henry, Ahcene Bouabdallah, Slim Kaddeche, Redouane Boussaa. Effect of rotation on the stability of side-heated buoyant convection between infinite horizontal walls. Physical Review Fluids, 2017, 2 (9), pp.093902. 10.1103/PhysRevFluids.2.093902 . hal-01724813

\section{HAL Id: hal-01724813 \\ https://hal.science/hal-01724813}

Submitted on 7 Mar 2018

HAL is a multi-disciplinary open access archive for the deposit and dissemination of scientific research documents, whether they are published or not. The documents may come from teaching and research institutions in France or abroad, or from public or private research centers.
L'archive ouverte pluridisciplinaire HAL, est destinée au dépôt et à la diffusion de documents scientifiques de niveau recherche, publiés ou non, émanant des établissements d'enseignement et de recherche français ou étrangers, des laboratoires publics ou privés. 


\title{
Effect of rotation on the stability of side-heated buoyant convection between infinite horizontal walls
}

\author{
A. Medelfef, ${ }^{1,2,{ }^{*}}$ D. Henry, ${ }^{2, \dagger}$ A. Bouabdallah, ${ }^{1}$ S. Kaddeche, ${ }^{3}$ and R. Boussaa ${ }^{1}$ \\ ${ }^{1}$ Laboratoire de Thermodynamique et Systèmes Energétiques, Faculté de Physique, Université des Sciences \\ et de la Technologie Houari Boumediène, BP 32, 16111 Bab Ezzouar, Alger, Algeria \\ ${ }^{2}$ Laboratoire de Mécanique des Fluides et d'Acoustique, CNRS, Université de Lyon, Ecole Centrale de Lyon, \\ Université Lyon 1, INSA de Lyon, ECL, 36 Avenue Guy de Collongue, 69134 Ecully Cedex, France \\ ${ }^{3}$ Unité de Recherche Matériaux, Mesures et Applications, Institut National des Sciences Appliquées et de \\ Technologie, BP 676, 1080 Tunis Cedex, Tunisia
}

(Received 21 April 2017; published 21 September 2017)

\begin{abstract}
This paper deals with buoyant convection generated by a horizontal gradient of temperature in an infinite fluid layer, which is known as Hadley circulation, and studies the effects induced by applying a rotation around the vertical axis. First, the basic flow profile with rotation is derived and the influence of the rotation is depicted: The original longitudinal velocity profile is decreased in intensity when rotation is applied and its structure is progressively changed, whereas a transverse velocity component is created, which increases with the rotation intensity, overcomes the longitudinal velocity, and eventually decreases. Different asymptotic behaviors for these profiles have also been highlighted. The stability of these flows is then studied. The effects of the Prandtl number, the Taylor number, and the thermal boundary conditions are highlighted for the three types of instability occurring in such a situation (shear, oscillatory, and Rayleigh instabilities). It is observed that they are all stabilized by the rotation and that the increase of the critical thresholds is accompanied by a spinning of the wave vector corresponding to a progressive change of the orientation of the marginal perturbation rolls. Energy budgets are finally used to analyze the instability mechanisms.
\end{abstract}

DOI: 10.1103/PhysRevFluids.2.093902

\section{INTRODUCTION}

Convection due to horizontal temperature differences has been the subject of intensive studies because of its implication in various natural, industrial and engineering problems such as planetary flows [1-4], and crystal growth processes [5]. These flows are known to be well described in the case of extended geometries by a simple model called Hadley circulation and corresponding to a cubic profile for the horizontal velocity along the confined vertical direction. This model was initially proposed by Hadley [1] to describe a single-cell flow generated by buoyancy, which could explain the atmospheric large-scale circulation between the equatorial hot regions and the cold poles.

In engineering and industrial processes, Hadley circulation is considered as a simplified model to describe the core of convective flows in extended side-heated cavities (known as shallow cavities) that are present in many industrial processes such as cooling of electronic components, nuclear reactors, habitations, and crystal growth technologies (particularly the horizontal Bridgman method) [5-7]. In the latter case, the appearance of hydrodynamic instabilities in the melt region dramatically affects the quality of the resulting crystals and it is thus important to study them to finally find a way to control these flows.

One of the first stability analyses of the Hadley circulation was conducted by Hart [8] and showed the coexistence of two types of instabilities that depend on the Prandtl number: a two-dimensional

\footnotetext{
*abs.medelfef@gmail.com

${ }^{\dagger}$ daniel.henry@ec-lyon.fr
} 
stationary shear instability corresponding to transverse rolls and due to the inflectional basic flow for weak Pr and an oscillatory instability corresponding to longitudinal rolls and resulting from the equilibrium between the stable temperature (density) stratification and the inertia of the flow for larger Pr.

Further stability studies were performed after this work. For example, Gill [7] explained the longitudinal oscillations by the mean of an asymptotic theoretical analysis based on small Prandtl numbers. Laure and Roux [9], Laure [10], and Gershuni et al. [11] deepened the analysis by considering the effects of the Prandtl number, the capillary forces, and the boundary conditions. In addition to the above-described instabilities, Gershuni et al. [11] pointed out the existence of longitudinal stationary thermal instabilities in the case of thermally conducting boundaries due to the unstable stratification of the temperature (density) profile along the horizontal walls. Pimputkar and Ostrach [12] and more recently Lappa [13] proposed extensive reviews of these situations.

The control of this type of flows was also the subject of extensive research activity. The magnetohydrodynamic flow has been investigated by Kaddeche et al. [14] and Henry et al. [15] for different magnetic-field orientations with respect to the mean flow direction. In the case of a vertical magnetic field, the work was recently further extended by Hudoba et al. [16] to take into account high-field intensities. Dridi et al. $[17,18]$, in a linear stability analysis, showed the effects of an acoustic streaming flow (generated by ultrasonic waves) on the different instabilities of Hadley circulation.

In addition to engineering and industrial processes, Hadley circulation was proposed to describe some geophysical and astrophysical flows. This was done, for example, by Stone [19,20] in studies related to different geophysical situations such as atmospheric Venusian circulation, tropical circulation, or even oceanic currents. It is known that these objects are in a perpetual rotation that strongly affects the flows dynamics by means of the noninertial Coriolis forces and thus the understanding of the dynamics and the stability of these rotating flows is important from a geophysicist's point of view.

Historically, the effect of the rotation about the vertical axis aligned with the gravity vector was examined in fluids heated from below in so-called Rayleigh-Bénard convection. One of the earlier studies of rotating convection was conducted by Jeffreys (see [21]) and it was reported that "the effect of rotation is to maintain stability." A considerable number of works have been published since then, such as the experimental and numerical study of Nakagawa and Frenzen [21], the famous stability study of Chandrasekhar [22] in which the rotation was included and emerged as a stabilizing factor, and the work on rapidly rotating convection of Zhang and Robert [23] where convection seems to be described at leading order by a thermal inviscid inertial wave when the Prandtl number is small.

Other works were also carried out in rotating differentially heated fluids as, for example, the experiments of Fultz [2] and Hide [3,4] in which the Hadley flow was observed before its bifurcation to other flow regimes such as Rossby waves and chaotic flows. Aristov and Frik [24] considered the effect of rotation on large-scale turbulence in a thin rotating fluid layer with a horizontal temperature gradient. Shvarts and Boudlal [25-27] carried out several stability studies in which the effect of rotation was examined. These studies investigated the effect of rotation on the stability of the advective flow and the behavior of finite-amplitude perturbations beyond the instability threshold for layers with solid boundaries [25] and with a free upper boundary [26] and finally in the case of thermocapillary convection for layers with two free boundaries in zero gravity. Furthermore, Chikulaev and Shvarts [28] studied the effect of rotation on the stability of the horizontally heated fluid layer with solid boundaries at small Prandtl numbers (typical values for liquid metals) and Knutova and Shvarts [29] examined the effect of rotation on the thermocapillary flow under microgravity conditions. In all these previous studies, however, the instability modes are either transverse (with $k_{x} \neq 0$ and $k_{y}=0$ ) or longitudinal (with $k_{x}=0$ and $k_{y} \neq 0$ ) rolls, which are, as will be shown in this paper, not the most unstable modes.

In the light of what has been done previously, we seek to examine the modification of the Hadley circulation when it is subjected to a rotation around the vertical axis and the change of its 


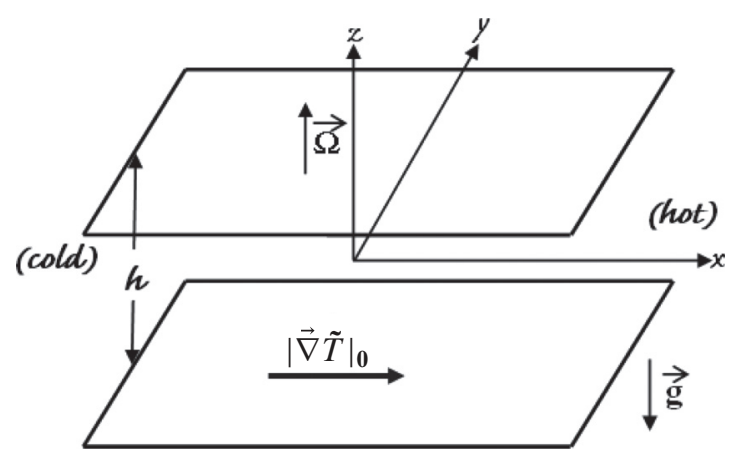

FIG. 1. Studied configuration and coordinates.

stability properties. The effects of the Prandtl number, the Taylor number, and the thermal boundary conditions are highlighted for the three types of instability occurring in such a situation.

\section{GOVERNING EQUATIONS AND BASIC FLOW}

\section{A. Governing equations}

We consider an incompressible fluid layer confined between two infinite rigid walls separated by a distance $h$ in the vertical $z$ direction. This layer is subjected to a constant horizontal temperature gradient $|\vec{\nabla} \tilde{T}|_{0}$ in the $x$ direction and is submitted to a rotation around the vertical $z$ axis (see Fig. 1). The equations governing the flow in such a situation have been derived, for example, by Chandrasekhar [22]. The fluid is assumed to be Newtonian with constant kinematic viscosity $v$ and thermal diffusivity $\kappa$. The Boussinesq approximation is generally applied, which means that the density is constant, except in the driving buoyancy term, where it is taken as a linear variation of the temperature $\rho=\rho_{0}\left[1-\gamma\left(\tilde{T}-\tilde{T}_{0}\right)\right]$, where $\gamma$ is the thermal expansion coefficient and $\tilde{T}_{0}$ is a reference temperature. The rotation is taken into account through the Coriolis force. As it is well known [22], the centrifugal force does not appear in the equations, as it can be expressed as a gradient and introduced in a modified pressure term.

Using $h, h^{2} / v, v / h, \rho v^{2} / h^{2}$, and $h|\vec{\nabla} \tilde{T}|_{0}$ as reference quantities for length, time, velocity, pressure, and temperature, respectively, the dimensionless governing equations are

$$
\begin{aligned}
\frac{\partial \vec{V}}{\partial t}+\vec{V} \cdot \vec{\nabla} \vec{V}+\sqrt{\operatorname{Ta}}\left(\vec{e}_{z} \times \vec{V}\right) & =-\vec{\nabla} P+\nabla^{2} \vec{V}+\operatorname{Gr} \Theta \vec{e}_{z} \\
\frac{\partial \Theta}{\partial t}+\vec{V} \cdot \vec{\nabla} \Theta & =\frac{1}{\operatorname{Pr}} \nabla^{2} \Theta, \\
\vec{\nabla} \cdot \vec{V} & =0
\end{aligned}
$$

where $\mathrm{Ta}=\frac{4 \Omega^{2} h^{4}}{\nu^{2}}$ is the Taylor number, $\mathrm{Gr}=\frac{g \gamma|\vec{\nabla} \tilde{T}|_{0} h^{4}}{\nu^{2}}$ is the Grashof number, and $\operatorname{Pr}=\frac{v}{\kappa}$ is the Prandtl number. The boundary conditions at the two horizontal rigid plates $(z= \pm 0.5)$ are no-slip conditions $(\vec{V}=0)$ and either perfectly conducting $(\Theta=x)$ or perfectly insulating $\left(\frac{d \Theta}{d z}=0\right)$ conditions.

\section{B. Basic flow profiles}

The layer is considered as infinitely long in both horizontal directions $x$ and $y$, but we also assume that there exist vertical boundaries located far from the center in both these directions $(x= \pm \infty$ and $y= \pm \infty$ ), which allow the flow to return and induce a zero flow rate in all the sections at fixed $x$ or at fixed $y$. In such a situation, a steady parallel flow solution depending only on the vertical $z$ 


\section{A. MEDELFEF et al.}

coordinate can be obtained. This steady-state flow is characterized by velocity profiles of the type $\left[U_{b}(z), V_{b}(z), 0\right]$, where $U_{b}(z)$ is the longitudinal component of the velocity originally created by the buoyancy force, and $V_{b}(z)$ is the transverse component created by the Coriolis effect. This transverse component must be equal to zero in the case without rotation where the usual Hadley circulation is retrieved. The temperature profile is given by $\Theta_{b}(x, z)=x+T_{b}(z)$, where the $x$ variation is related to the imposed temperature gradient and $T_{b}(z)$ represents the vertical profile of temperature to be determined.

By introducing the basic flow quantities $U_{b}(z), V_{b}(z)$, and $\Theta_{b}(x, z)$ and taking the curl of the momentum conservation equation in order to eliminate the pressure term, Eqs. (1)-(3) lead to the ordinary differential equations

$$
\begin{aligned}
& \frac{d^{3} U_{b}}{d z^{3}}+\sqrt{\mathrm{Ta}} \frac{d V_{b}}{d z}=\mathrm{Gr}, \\
& \frac{d^{3} V_{b}}{d z^{3}}-\sqrt{\mathrm{Ta}} \frac{d U_{b}}{d z}=0
\end{aligned}
$$

for the velocity profile and

$$
\frac{d^{2} T_{b}}{d z^{2}}-\operatorname{Pr} U_{b}=0
$$

for the temperature profile. The boundary conditions associated with these equations are $U_{b}(z=$ $\pm 0.5)=V_{b}(z= \pm 0.5)=0$ ensuring the no-slip boundary condition, $U_{b}(z=0)=V_{b}(z=0)=0$ ensuring the zero flow rate for profiles that are symmetric with respect to the center $z=0$, and either $T_{b}(z= \pm 0.5)=0$ for perfectly conducting walls or $\left(\frac{d T_{b}}{d z}\right)(z= \pm 0.5)=0$ for perfectly insulating walls.

Equations (4a) and (4b) are solved by introducing a complex velocity profile defined as $\Phi(z)=$ $U_{b}(z)+i V_{b}(z)$, which leads to a unique and simple third-order differential equation

$$
\frac{d^{3} \Phi}{d z^{3}}-i \sqrt{\mathrm{Ta}} \frac{d \Phi}{d z}=\mathrm{Gr}
$$

associated with the boundary conditions $\Phi(z= \pm 0.5)=0$ and $\Phi(z=0)=0$. By solving Eq. (6), we obtain the complex velocity profile

$$
\begin{aligned}
\Phi(z) & =\frac{i \mathrm{Gr}}{2 \sqrt{\mathrm{Ta}}}\left(2 z-\frac{\exp \left(\frac{(i+1) \mathrm{Ta}^{1 / 4}}{\sqrt{2}} z\right)-\exp \left(-\frac{(i+1) \mathrm{Ta}^{1 / 4}}{\sqrt{2}} z\right)}{\exp \left(\frac{(i+1) \mathrm{Ta}^{1 / 4}}{2 \sqrt{2}}\right)-\exp \left(-\frac{(i+1) \mathrm{Ta}^{1 / 4}}{2 \sqrt{2}}\right)}\right) \\
& =\frac{i \mathrm{Gr}}{2 \sqrt{\mathrm{Ta}}}\left(2 z-\frac{\sinh \left(\frac{(i+1) \mathrm{Ta}^{1 / 4}}{\sqrt{2}} z\right)}{\sinh \left(\frac{(i+1) \mathrm{Ta}^{1 / 4}}{2 \sqrt{2}}\right)}\right) .
\end{aligned}
$$

Note that this profile is similar to that given by Chikulaev and Shvarts [28]. Finally, the velocity components $U_{b}(z)$ and $V_{b}(z)$ can be deduced by separating the real and imaginary parts of the complex function $\Phi(z)$, which gives

$$
\begin{aligned}
& U_{b}(z)=\frac{\mathrm{Gr}}{2 \sqrt{\mathrm{Ta}}}\left(\frac{\alpha \cosh \left(\frac{\mathrm{Ta}^{1 / 4}}{\sqrt{2}} z\right) \sin \left(\frac{\mathrm{Ta}^{1 / 4}}{\sqrt{2}} z\right)-\beta \sinh \left(\frac{\mathrm{Ta}^{1 / 4}}{\sqrt{2}} z\right) \cos \left(\frac{\mathrm{Ta}^{1 / 4}}{\sqrt{2}} z\right)}{\alpha^{2}+\beta^{2}}\right), \\
& V_{b}(z)=\frac{\mathrm{Gr}}{2 \sqrt{\mathrm{Ta}}}\left(2 z-\frac{\alpha \sinh \left(\frac{\mathrm{Ta}^{1 / 4}}{\sqrt{2}} z\right) \cos \left(\frac{\mathrm{Ta}^{1 / 4}}{\sqrt{2}} z\right)+\beta \cosh \left(\frac{\mathrm{Ta}^{1 / 4}}{\sqrt{2}} z\right) \sin \left(\frac{\mathrm{Ta}^{1 / 4}}{\sqrt{2}} z\right)}{\alpha^{2}+\beta^{2}}\right),
\end{aligned}
$$



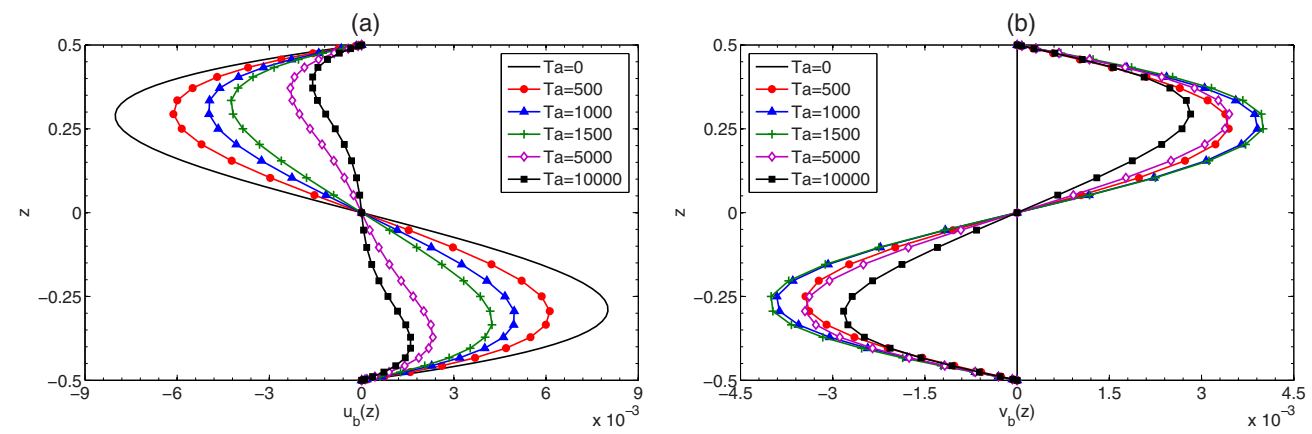

FIG. 2. Effect of the Taylor number Ta on (a) the longitudinal $u_{b}(z)=U_{b}(z) / \mathrm{Gr}$ and (b) the transverse $v_{b}(z)=V_{b}(z) / \mathrm{Gr}$ velocity profiles.

with

$$
\begin{aligned}
& \alpha(\mathrm{Ta})=\sinh \left(\frac{\mathrm{Ta}^{1 / 4}}{2 \sqrt{2}}\right) \cos \left(\frac{\mathrm{Ta}^{1 / 4}}{2 \sqrt{2}}\right), \\
& \beta(\mathrm{Ta})=\cosh \left(\frac{\mathrm{Ta}^{1 / 4}}{2 \sqrt{2}}\right) \sin \left(\frac{\mathrm{Ta}^{1 / 4}}{2 \sqrt{2}}\right) .
\end{aligned}
$$

It is interesting to evaluate the asymptotic expressions of the velocities for $\mathrm{Ta} \rightarrow 0$. The series expansion of the complex expression (7b) as a function of Ta leads to

$$
\Phi(z)=\operatorname{Gr}\left[\left(\frac{z^{3}}{6}-\frac{z}{24}\right)-\operatorname{Ta} Q(z)+i \sqrt{\mathrm{Ta}} H(z)\right]+O\left(\mathrm{Ta}^{3 / 2}\right),
$$

with

$$
\begin{aligned}
& Q(z)=\frac{1}{5040} z^{7}-\frac{1}{2880} z^{5}+\frac{7}{34560} z^{3}-\frac{31}{967680} z \\
& H(z)=\frac{1}{120} z^{5}-\frac{1}{144} z^{3}+\frac{7}{5760} z .
\end{aligned}
$$

From Eq. (9), we can first check that the limit of $\Phi(z)$ when Ta $\rightarrow 0$ (i.e., without rotation) is the well-known Hadley profile obtained, for example, by Hart [8]:

$$
U_{H}=\operatorname{Gr}\left(\frac{z^{3}}{6}-\frac{z}{24}\right)
$$

Equation (9) also indicates that, when rotation is applied, the departure of the longitudinal velocity from the Hadley profile occurs as a seventh-order polynomial given by $Q(z)$ and is proportional to $\mathrm{Ta}$, whereas the initiation of the transverse velocity occurs as a fifth-order polynomial given by $H(z)$ and is proportional to $\sqrt{\mathrm{Ta}}$.

Figures 2(a) and 2(b) show the effect of the Taylor number on the velocity profiles $u_{b}(z)=$ $U_{b}(z) / \mathrm{Gr}$ and $v_{b}(z)=V_{b}(z) / \mathrm{Gr}$ obtained from Eqs. (8a) and (8b), respectively. In the case without rotation $(\mathrm{Ta}=0)$, as expected, the profile is purely longitudinal with $U_{b}(z)$ corresponding to the Hadley cubical profile given by Eq. (11). When the Taylor number is increased, the dynamics of the flow is modified by the Coriolis term, which induces a deviation of the flow to the right, thus creating transverse velocities ( $V_{b}<0$ when $U_{b}>0$ and $V_{b}>0$ when $U_{b}<0$, in our coordinates). The transverse velocities will increase for increasing Taylor numbers up to Ta $\simeq 1500$, while the longitudinal velocities decrease due to the action of the Coriolis force on these transverse velocities. Note that at $\mathrm{Ta}=1500$, the velocity components have similar intensities. As the Taylor number is further increased, both velocity components will finally decrease. Such a behavior was already 


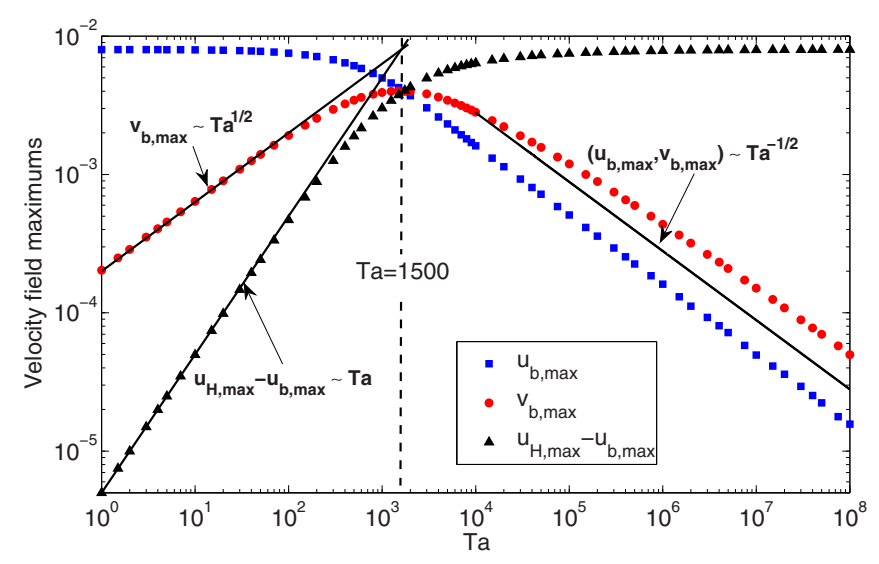

FIG. 3. Maximum velocity components $u_{b, \max }=U_{b, \max } / \mathrm{Gr}$ (squares), $v_{b, \max }=V_{b, \max } / \mathrm{Gr}$ (circles), and $u_{H, \max }-u_{b, \max }$ (triangles) as a function of the Taylor number Ta.

observed in rotating flows by Shvarts and Boudlal [26] and Mehdizadeh and Oberlack [30], for example.

More precision on the variations of the velocities intensities when rotation is applied can be obtained from Fig. 3 where the maximums of the velocity components $u_{b}(z)$ and $v_{b}(z)$ are given as a function of the Taylor number. The initial increase of $v_{b, \max }$ occurs as $\mathrm{Ta}^{1 / 2}$, while that of $u_{H, \max }-u_{b, \max }$ occurs as Ta, as given by (9). The maximum of $v_{b, \max }$ is really close to Ta $=1500$ and very close to the intersection of the curves of $u_{b, \max }$ and $v_{b, \max }$. The decrease of the two maximums $u_{b, \max }$ and $v_{b, \max }$ when the Taylor number is further increased seems to follow a $\mathrm{Ta}^{-1 / 2}$ law, more clearly for the longitudinal component than for the transverse component.

The limit of the velocity profiles for strong rotation $(\mathrm{Ta} \rightarrow \infty)$ can be studied analytically. First, for $\mathrm{Ta} \rightarrow \infty$, by neglecting an exponential term in the denominator, the expression (7a) can be simplified and written as

$$
\Phi_{\infty}(z) \approx \frac{i \mathrm{Gr}}{2 \sqrt{\mathrm{Ta}}}\left[2 z-\exp \left(-\frac{(i+1) \mathrm{Ta}^{1 / 4}}{\sqrt{2}}(0.5-z)\right)+\exp \left(-\frac{(i+1) \mathrm{Ta}^{1 / 4}}{\sqrt{2}}(0.5+z)\right)\right]
$$

For large Ta, the first exponential in (12) is nonzero close to $z=0.5$, whereas the second exponential is nonzero close to $z=-0.5$, so we can split the problem and define

$$
\begin{gathered}
\Phi_{\infty}(-0.5 \rightarrow 0) \approx \frac{i \mathrm{Gr}}{2 \sqrt{\mathrm{Ta}}}\left[2 z+\exp \left(-\frac{(i+1) \mathrm{Ta}^{1 / 4}}{\sqrt{2}}(0.5+z)\right)\right], \\
\Phi_{\infty}(0 \rightarrow 0.5) \approx \frac{i \mathrm{Gr}}{2 \sqrt{\mathrm{Ta}}}\left[2 z-\exp \left(-\frac{(i+1) \mathrm{Ta}^{1 / 4}}{\sqrt{2}}(0.5-z)\right)\right] .
\end{gathered}
$$

As we know that the profiles are symmetric with respect to $z=0$, the study can focus on one of these expressions. We can show that, in these expressions, the exponentials are zero everywhere for large $\mathrm{Ta}$, except close to the boundaries. Moreover, they have finite maximum values

$$
\pm \exp (-3 \pi / 4) \cos (3 \pi / 4) \quad \text { at } z=\mp\left(0.5-\frac{3 \sqrt{2} \pi}{4 T a^{1 / 4}}\right)
$$

for their real part, i.e., for $V_{b}$, and

$$
\pm \exp (-\pi / 4) \sin (-\pi / 4) \quad \text { at } z=\mp\left(0.5-\frac{\sqrt{2} \pi}{4 T a^{1 / 4}}\right)
$$


(a)

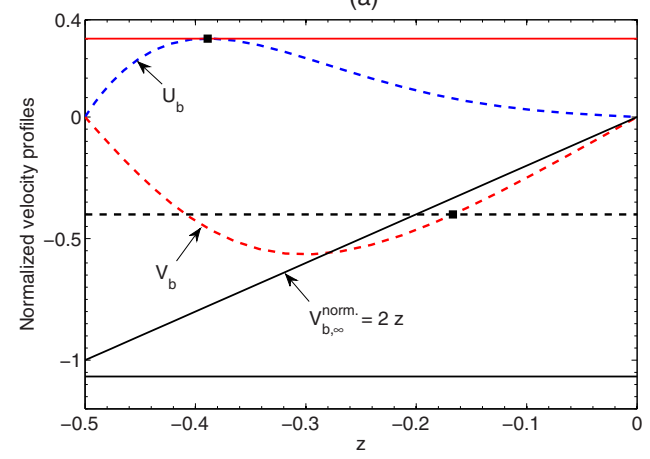

(b)

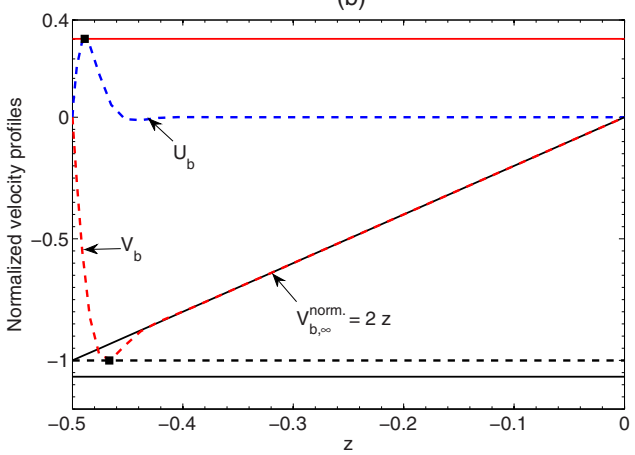

FIG. 4. Longitudinal $U_{b}(z)$ and transverse $V_{b}(z)$ velocity profiles in the bottom half of the cavity $(-0.5 \leqslant$ $z \leqslant 0)$. The velocities are divided by the prefactor $\mathrm{Gr} / 2 \sqrt{\mathrm{Ta}}$. They are given for (a) $\mathrm{Ta}=10^{4}$ and $(\mathrm{b}) \mathrm{Ta}=10^{8}$ (positive profiles for $U_{b}$ in blue dashed lines and negative profiles for $V_{b}$ in red dashed lines). The asymptotic values for the maximums are given as horizontal lines for $V_{b}$ from Eq. (17a) (black dashed lines), $V_{b}$ from Eq. (17b) (black solid lines), and $U_{b}$ from Eq. (16) (red solid lines). The positions of these maximums [given in Eqs. (14) and (15) for $V_{b}$ and $U_{b}$, respectively] are plotted as black squares along these lines.

for their imaginary part, i.e., for $U_{b}$. In the expressions (13a) and (13b), only the prefactor will then depend on Ta. This indicates that both $U_{b}$ and $V_{b}$ will eventually decrease at large Ta as $\mathrm{Ta}^{-1 / 2}$, as was observed in Fig. 3. More precisely, the maximums of $U_{b}$ (only given by the imaginary part of the exponentials) occur close to the boundaries, at the positions given in (15), and will correspond to

$$
U_{b, \infty, \max }= \pm \frac{\mathrm{Gr}}{2 \sqrt{\mathrm{Ta}}} \exp (-\pi / 4) \sin (\pi / 4)
$$

whereas $U_{b, \infty}$ is close to zero in most of the cavity. In contrast, the maximums of $V_{b}$ are those of the linear function $2 z$ plus the real part of the exponential. Only at very large Ta can they approximately occur at the position given in (14) and be given by

$$
\begin{aligned}
V_{b, \infty, \max } & \approx \pm \frac{\mathrm{Gr}}{2 \sqrt{\mathrm{Ta}}}\left[-1+\frac{3 \sqrt{2} \pi}{2 \mathrm{Ta}^{1 / 4}}-\exp (-3 \pi / 4) \sin (\pi / 4)\right] \\
& \approx \pm \frac{G r}{2 \sqrt{\mathrm{Ta}}}[-1-\exp (-3 \pi / 4) \sin (\pi / 4)] .
\end{aligned}
$$

The asymptotic expression (17b) is valid at very large Ta when the term $3 \sqrt{2} \pi / 2 \mathrm{Ta}^{1 / 4}$ in (17a) becomes negligible with respect to 1 .

Note that $V_{b, \infty}$ is close to the linear function $z \frac{\mathrm{Gr}}{\sqrt{\mathrm{Ta}}}$ in most of the cavity. These asymptotic behaviors are confirmed by the results shown in Fig. 4, where the velocity profiles in the bottom half of the cavity, normalized by $\mathrm{Gr} /(2 \sqrt{\mathrm{Ta}})$, are given for $\mathrm{Ta}=10^{4}$ and $10^{8}$. The maximum of the normalized longitudinal velocity is in very good agreement with both the asymptotic value given by (16) and the position given in (15) for the two values of Ta. This indicates that the asymptotic behavior for $U_{b}$ is already reached for $\mathrm{Ta}=10^{4}$. In contrast, the normalized transverse velocity is far from the asymptotic behavior at $\mathrm{Ta}=10^{4}$. For $\mathrm{Ta}=10^{8}$, the agreement with the asymptotic behavior is far better: a linear profile valid in most of the cavity, the position of the maximum given by the expression in (14), and the value of this maximum given by (17a). However, higher values of $\mathrm{Ta}$, as $\mathrm{Ta}=10^{12}$, are necessary to reach the true asymptotic maximum given by $(17 \mathrm{~b})$. 
(a)

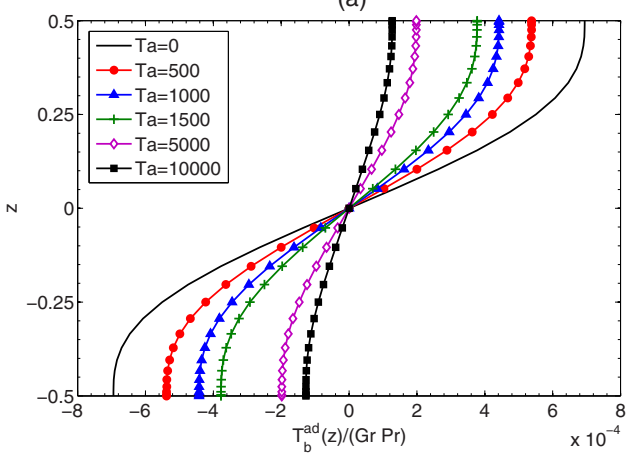

(b)

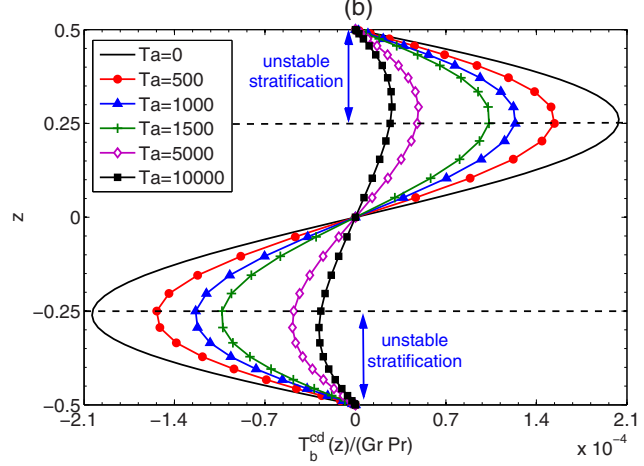

FIG. 5. Effect of the Taylor number Ta on the vertical temperature profiles $T_{b}(z) / \mathrm{Gr} \operatorname{Pr}$ for (a) thermally insulating walls and (b) thermally conducting walls.

The vertical temperature profiles $T_{b}(z)$ are obtained by integration of Eq. (2), with the use of the expression (8a) for $U_{b}$. We get

$$
T_{b}(z)=-\frac{\mathrm{Gr} \operatorname{Pr}}{2 \mathrm{Ta}}\left(\frac{\alpha \sinh \left(\frac{\mathrm{Ta}^{1 / 4}}{\sqrt{2}} z\right) \cos \left(\frac{\mathrm{Ta}^{1 / 4}}{\sqrt{2}} z\right)+\beta \cosh \left(\frac{\mathrm{Ta}^{1 / 4}}{\sqrt{2}} z\right) \sin \left(\frac{\mathrm{Ta}^{1 / 4}}{\sqrt{2}} z\right)}{\alpha^{2}+\beta^{2}}\right)+C_{1} z+C_{2} .
$$

The integration constant $C_{1}$ is obtained by applying the thermal boundary conditions. For insulating and conducting walls, we get

$$
\begin{aligned}
C_{1}^{\mathrm{ad}}= & \frac{\operatorname{Pr} \mathrm{Gr}}{2 \sqrt{2} \mathrm{Ta}^{3 / 4}} \frac{1}{\alpha^{2}+\beta^{2}}\left[(\alpha+\beta) \cosh \left(\frac{\mathrm{Ta}^{1 / 4}}{2 \sqrt{2}}\right) \cos \left(\frac{\mathrm{Ta}^{1 / 4}}{2 \sqrt{2}}\right)\right. \\
& \left.-(\alpha-\beta) \sinh \left(\frac{\mathrm{Ta}^{1 / 4}}{2 \sqrt{2}}\right) \sin \left(\frac{\mathrm{Ta}^{1 / 4}}{2 \sqrt{2}}\right)\right]
\end{aligned}
$$

and

$$
C_{1}^{\mathrm{cd}}=\frac{\mathrm{Gr} \operatorname{Pr}}{\mathrm{Ta}},
$$

respectively. Note that the constant $C_{2}$ vanishes in both cases because of the symmetry of the problem. The series expansion of Eq. (18) for Ta close to zero gives

$$
T_{b}^{\mathrm{ad}}(z)=\operatorname{Gr} \operatorname{Pr}\left(\frac{z^{5}}{120}-\frac{z^{3}}{144}+\frac{z}{384}\right)+O(\mathrm{Ta})
$$

and

$$
T_{b}^{\mathrm{cd}}(z)=\operatorname{Gr} \operatorname{Pr}\left(\frac{z^{5}}{120}-\frac{z^{3}}{144}+\frac{7 z}{5760}\right)+O(\mathrm{Ta})
$$

for insulating and conducting walls, respectively. For $\mathrm{Ta} \rightarrow 0$, we then obtain the profiles given by Hart [8] in the case without rotation.

The effect of the rotation on the temperature profiles is reported in Fig. 5 for both thermal boundary conditions (insulating walls [Fig. 5(a)] and conducting walls [Fig. 5(b)]). We observe a progressive suppression of the vertical temperature gradients when the rotation is increased: This is connected to the decrease of the longitudinal velocity component $U_{b}(z)$ responsible for these vertical temperature gradients. One should notice the existence of unstable thermal stratification regions near the walls in the case of conducting boundary conditions, which will be at the origin of thermal instabilities (Rayleigh modes). Note finally that for all these basic vertical stationary profiles 
[Eqs. (8a) and (8b) for the velocities and (18) for the temperature], the Grashof and the Prandtl numbers only appear as multiplicative factors, which makes the quantities $\left(u_{b}, v_{b}\right)=\left(U_{b}, V_{b}\right) / \mathrm{Gr}$ and $T_{b} / \mathrm{Gr}$ Pr only dependent on the Taylor number.

\section{LINEAR STABILITY ANALYSIS}

The stability analysis of the basic flow has been performed by means of a spectral collocation method. The general solution of the problem is expressed as $\vec{V}=\vec{V}_{b}+\vec{v}, \Theta=\Theta_{b}+\theta$, and $P=$ $P_{b}+p$, where $(\vec{v}, \theta, p)$ are infinitesimal perturbations. This solution is introduced in the system (1)-(3) and after linearization with respect to the perturbations (second-order terms neglected), we obtain the governing system for these perturbations:

$$
\begin{gathered}
\frac{\partial \vec{v}}{\partial t}+\vec{v} \cdot \vec{\nabla} \vec{V}_{b}+\vec{V}_{b} \cdot \vec{\nabla} \vec{v}+\sqrt{\operatorname{Ta}}\left(\vec{e}_{z} \times \vec{v}\right)=-\vec{\nabla} p+\nabla^{2} \vec{v}+\operatorname{Gr} \theta \vec{e}_{z}, \\
\frac{\partial \theta}{\partial t}+\vec{V}_{b} \cdot \vec{\nabla} \theta+\vec{v} \cdot \vec{\nabla} \Theta_{b}=\frac{1}{\operatorname{Pr}} \nabla^{2} \theta, \\
\vec{\nabla} \cdot \vec{v}=0 .
\end{gathered}
$$

In an extended layer, the perturbations can be expressed as normal modes in both the $x$ and $y$ directions,

$$
(\vec{v}, \theta, p)=(\vec{v}, \theta, p)(z) e^{s t+i\left(k_{x} x+k_{y} y\right)},
$$

where $k_{x}$ and $k_{y}$ are real wave numbers in the longitudinal, $x$, and transverse, $y$, directions, respectively, defining a wave vector $\vec{k}$, and $s=\sigma+i \omega$ is a complex eigenvalue. The real part $\sigma$ represents an amplification rate and the imaginary part $\omega$ an oscillation frequency. Only boundary conditions at $z= \pm 0.5$ are needed to solve this system and they are given by $u=v=w=0$ and $\theta=0$ for perfectly conducting walls or $d \theta / d z=0$ for perfectly insulating walls. A generalized eigenvalue problem is obtained: $L X=(\sigma+i \omega) M X$, where $X=[u(z), v(z), w(z), p(z), \theta(z)], L$ is a linear operator depending on $k_{x}, k_{y}, \mathrm{Pr}, \mathrm{Gr}$, and $\mathrm{Ta}$, and $M$ is a constant linear operator. This eigenvalue problem is solved numerically using a Chebychev collocation method. From the thresholds $\mathrm{Gr}_{0}\left(\mathrm{Pr}, \mathrm{Ta}, k_{x}, k_{y}\right)$ (values of $\mathrm{Gr}$ for which an eigenvalue has a real part equal to zero, whereas all the other eigenvalues have negative real parts), the critical Grashof number $\mathrm{Gr}_{c}$ can be obtained after minimization along the wave numbers $k_{x}$ and $k_{y}$. These critical Grashof numbers were calculated by a discretization of the physical domain using 31 collocation points, which is sufficient for an accurate determination of the thresholds and their characteristics.

The minimization procedure is performed by using the Nelder-Mead simplex method described by Lagarias et al. [31]. This technique finds the local minimum of a several-variable scalar function starting from an initial estimate of these variables (here the wave numbers). The simplex of the Nelder-Mead method, for a two-variable function $\left(k_{x}\right.$ and $\left.k_{y}\right)$, is a triangle for which the values of the function at its three vertices are compared. The worst vertex (for which the value of the function is the largest one) is then rejected and replaced with a new vertex. The objective is then to reduce the size of the triangle until the convergence criterion is reached. For more details on the method and its practical use, the reader can consult Ref. [32]. Minimization is carried out along $\left(k_{x}, 0\right)$ for the transverse modes, along $\left(0, k_{y}\right)$ for the longitudinal modes, and along the couple $\left(k_{x}, k_{y}\right)$ for the oblique modes. Finally, these modes are either steady or oscillatory, depending on whether the imaginary part of the eigenvalue is zero or nonzero, respectively. Our results are presented in the following section.

\section{STABILITY RESULTS}

The stability of the flow was first studied in the case without rotation $(\mathrm{Ta}=0)$ in order to compare with the results of Laure [10]. The critical Grashof numbers $\mathrm{Gr}_{c}$ corresponding to the appearance of 
TABLE I. Critical thresholds $\mathrm{Gr}_{c}$ corresponding to the case without rotation $(\mathrm{Ta}=0)$ and with thermally insulating walls.

\begin{tabular}{lccr}
\hline \hline $\operatorname{Pr}$ & $\mathrm{Gr}_{c}$ & $\omega_{c}$ & Ref. [10] \\
\hline 0.001 & 7944.26 & 0 & 7942 \\
0.01 & 8165.19 & 0 & 8168 \\
0.05 & 8446.33 & 36.56 & 8445 \\
0.1 & 7342.14 & 38.96 & 7342 \\
\hline \hline
\end{tabular}

the first unstable mode for some Pr numbers and for Ta $=0$ are given in Table I. Good agreement with the results of Laure [10] is found. Note that, in the case without rotation, it was shown that the critical modes are either transverse modes $\left(k_{y}=0\right)$ or longitudinal modes $\left(k_{x}=0\right)$, so the oblique modes are never the most unstable (see $[9,10]$, for example). This was also verified during this study.

At this stage, it is convenient to present the main instabilities existing for $\mathrm{Ta}=0$ and to locate them in the space of the parameters $\left(\mathrm{Gr}_{c}\right.$ and $\left.\mathrm{Pr}\right)$. The critical thresholds for these instabilities are given as a function of Pr in Figs. 6(a) and 6(b) for thermally insulating and thermally conducting walls, respectively. A stationary transverse shear mode is found for the lowest values of Pr: The corresponding thresholds initially remain practically constant while the Prandtl number is increased up to values between 0.01 and 0.1 (low-Pr asymptotic domain), before a quite rapid growth phase. An oscillatory longitudinal mode is then found for larger values of Pr: The critical curve decreases as Pr is increased, intersects the shear mode critical curve, and then reaches a minimum before undergoing a rapid growth phase. These two modes are obtained for both thermal boundary conditions, with only changes in the exact variation of the critical curves with Pr. In contrast, the stationary longitudinal Rayleigh modes are only obtained for thermally conducting walls due to the presence of unstable stratification along these walls [see Fig. 5(b)]: In the range of $\operatorname{Pr}$ studied ( $\operatorname{Pr} \leqslant 10)$, the critical curve decreases when the Prandtl number is increased, so this instability becomes dominant for $\operatorname{Pr}>0.45$.

When rotation is applied, it is expected to have an influence on the critical modes and on their thresholds, but also on the spatial orientation of these modes. The thresholds for oblique orientations of the modes then have to be calculated and compared with those obtained for the usual transverse and longitudinal orientations, in order to determine the true critical thresholds. These comparisons are presented in Figs. 7(a) and 7(b), where the variations of the critical stability curves with the Taylor number Ta are given for different types of instabilities and different orientations (wave vector $\vec{k}$ along an axis or oblique). Some numerical values for the thresholds are also given in Table II. We can clearly observe that, when the Taylor number is increased, the thresholds of these instabilities are shifted to higher values for all cases, which indicates a stabilization effect. This stabilization
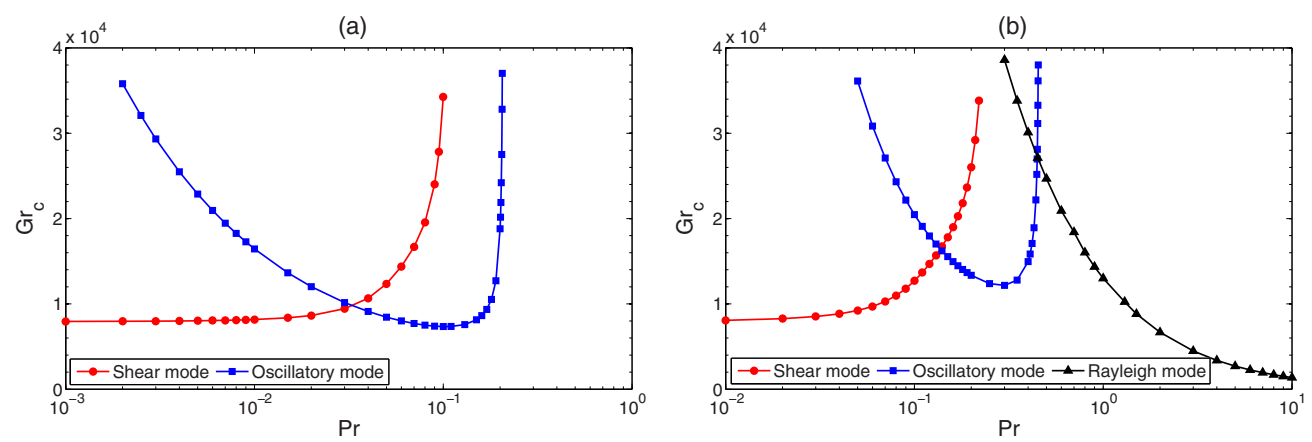

FIG. 6. Instability thresholds $\mathrm{Gr}_{c}$ as a function of Pr for the main modes at $\mathrm{Ta}=0$ for (a) thermally insulating boundaries and (b) thermally conducting boundaries. 
(a)

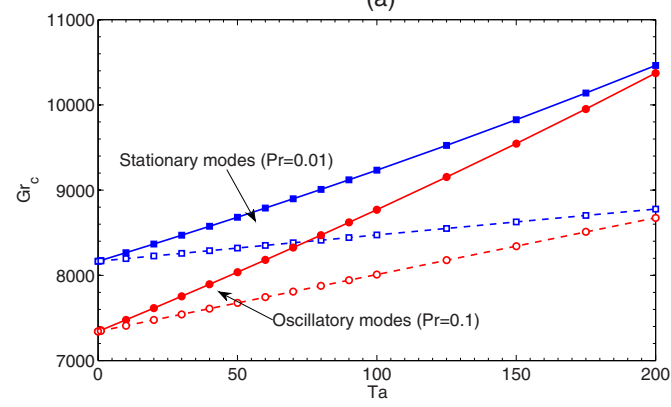

(b)

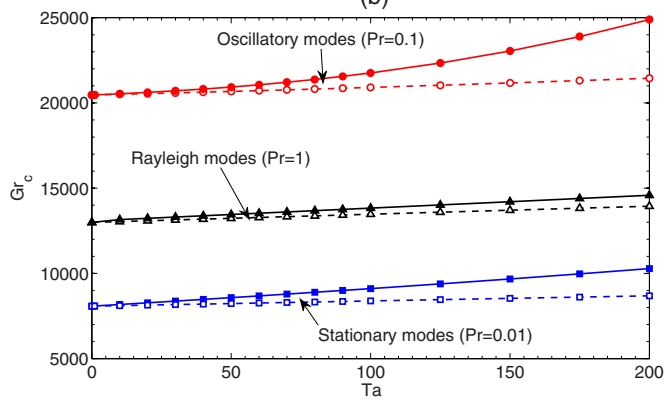

FIG. 7. Variation of the critical thresholds $\mathrm{Gr}_{c}$ as a function of the Taylor number Ta for different instability modes for (a) thermally insulating boundaries and (b) thermally conducting boundaries. The oblique modes are given as dashed lines, while the transverse and longitudinal modes are given as solid lines.

effect, however, is more important in the case of the fixed orientations (transverse or longitudinal, depending on the instability), indicating thus that, in any case, oblique modes become the dominant instabilities when rotation is applied. Note that the Rayleigh instability can change its temporal behavior from stationary to oscillatory in the case with rotation, while the other instabilities retain their usual temporal behavior.

\section{A. Shear instability}

The effect of the rotation on the thresholds of the shear instability is studied first. The evolution with Ta of the critical Grashof number $\mathrm{Gr}_{c}$ corresponding to stationary oblique modes is plotted in

TABLE II. Critical thresholds obtained for pure convection $(\mathrm{Ta}=0)$ and convection submitted to rotation $(\mathrm{Ta}=100)$. Here STM, OLM, RLM, SOM, OOM, and ROM stand for stationary transverse, oscillatory longitudinal, Rayleigh longitudinal, stationary oblique, oscillatory oblique, and Rayleigh oblique modes, respectively.

\begin{tabular}{|c|c|c|c|c|c|}
\hline $\mathrm{Ta}$ & Characteristics & $\mathrm{Gr}_{c}$ & $k_{x, c}$ & $k_{y, c}$ & $\omega_{c}$ \\
\hline \multicolumn{6}{|c|}{ Insulating walls } \\
\hline 0 & $\operatorname{STM}(\operatorname{Pr}=0.01)$ & 8165.19 & 2.68 & 0 & 0 \\
\hline 0 & $\operatorname{OLM}(\operatorname{Pr}=0.1)$ & 7342.14 & 0 & 1.02 & 38.96 \\
\hline 100 & $\operatorname{STM}(\operatorname{Pr}=0.01)$ & 9234.09 & 2.61 & 0 & 0 \\
\hline 100 & $\operatorname{SOM}(\operatorname{Pr}=0.01)$ & 8474.03 & 2.52 & -0.97 & 0 \\
\hline 100 & $\operatorname{OLM}(\operatorname{Pr}=0.1)$ & 8770.32 & 0 & 1.07 & 46.65 \\
\hline 100 & $\mathrm{OOM}(\operatorname{Pr}=0.1)$ & 8009.86 & 0.20 & 1.04 & 42.19 \\
\hline \multicolumn{6}{|c|}{ Conducting walls } \\
\hline 0 & $\mathrm{STM}(\operatorname{Pr}=0.01)$ & 8076.92 & 2.68 & 0 & 0 \\
\hline 0 & OLM $(\operatorname{Pr}=0.1)$ & 20466.78 & 0 & 2.01 & 126.46 \\
\hline 0 & $\operatorname{RLM}(\operatorname{Pr}=0.7)$ & 18152.24 & 0 & 8.23 & 0 \\
\hline 100 & $\operatorname{STM}(\operatorname{Pr}=0.01)$ & 9106.81 & 2.61 & 0 & 0 \\
\hline 100 & $\operatorname{SOM}(\operatorname{Pr}=0.01)$ & 8383.69 & 2.52 & -0.98 & 0 \\
\hline 100 & $\mathrm{OLM}(\mathrm{Pr}=0.1)$ & 21759.98 & 0 & 1.84 & 126.34 \\
\hline 100 & $\mathrm{OOM}(\mathrm{Pr}=0.1)$ & 20911.98 & 0.23 & 1.97 & 125.46 \\
\hline 100 & $\operatorname{RLM}(\operatorname{Pr}=0.7)$ & 19405.55 & 0 & 8.34 & 215.05 \\
\hline 100 & $\operatorname{ROM}(\operatorname{Pr}=0.7)$ & 18794.52 & 1.82 & 8.01 & 0 \\
\hline
\end{tabular}



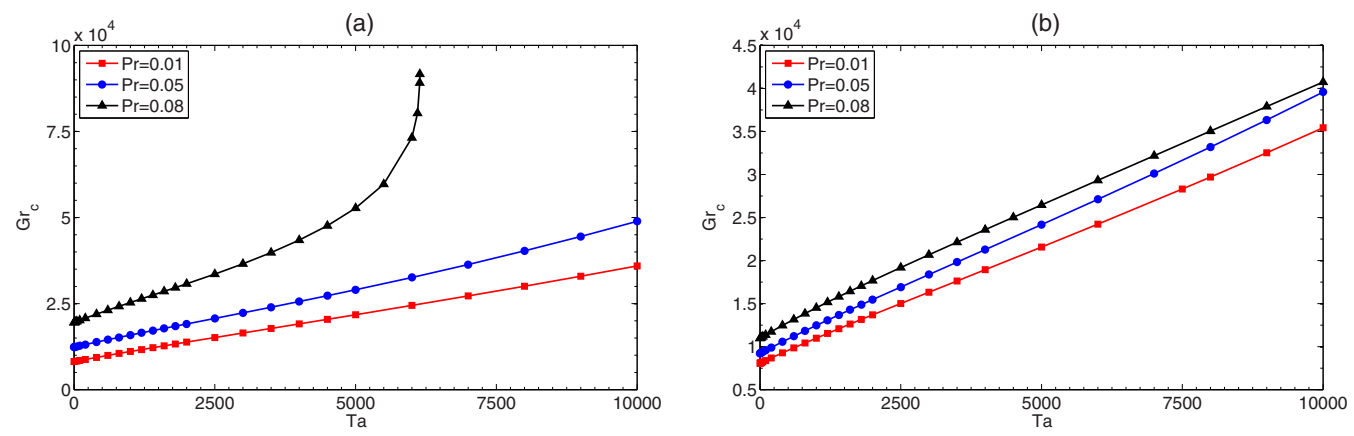

FIG. 8. Variation of the thresholds $\mathrm{Gr}_{c}$ for the stationary shear instability as a function of the Taylor number Ta for different Prandtl numbers Pr for (a) thermally insulating boundaries and (b) thermally conducting boundaries.

Fig. 8. Three values of the Prandtl number Pr are considered $(0.01,0.05$, and 0.08$)$ and the results are given for both insulating walls [Fig. 8(a)] and conducting walls [Fig. 8(b)].

In the range of Ta studied (Ta $\leqslant 10000$ ), for both thermal boundary conditions [Figs. 8(a) and $8(\mathrm{~b})$ ], the thresholds increase linearly with Ta for the smallest values of the Prandtl number $(\mathrm{Pr}=$ 0.01 and 0.05 for insulating boundaries and $\mathrm{Pr}=0.01,0.05$ and 0.08 for conducting boundaries) in the asymptotic low-Pr range (domain of constant threshold in Fig. 6). In contrast, at $\operatorname{Pr}=0.08$ for insulating boundaries [Fig. 8(a)], we observe an initial linear variation of the thresholds with Ta, followed by a rapid increase. Further calculations performed for $\operatorname{Pr}=0.09$ and 0.1 in the insulating case show that the Ta range of linear variation shrinks when $\mathrm{Pr}$ is increased, indicating a more effective stabilization of these shear modes by rotation.

The effect of the rotation on the critical wave vector $\vec{k}_{c}$ can be described through the variation with Ta of its norm (defined as $\left|\vec{k}_{c}\right|=\sqrt{k_{x, c}^{2}+k_{y, c}^{2}}$ ) and its orientation in the $x y$ plane. In practice, we follow the normal to the wave vector (i.e., the axis of the generated counterrotating rolls perturbation) with respect to the $x$ axis and define the angle $\phi=\arctan \left(-\frac{k_{x, c}}{k_{y, c}}\right)\left(\phi\right.$ is equal to $90^{\circ}$ for the transverse modes and $0^{\circ}$ for the longitudinal modes). The action of the rotation on the norm of the wave number is reported in Figs. 9(a) (insulating walls) and 9(b) (conducting walls) and its action on $\phi$ in Fig. 10 (conducting walls). For Ta $=0$, the wave vector $\vec{k}_{c}$ for the stationary transverse shear modes

(a)

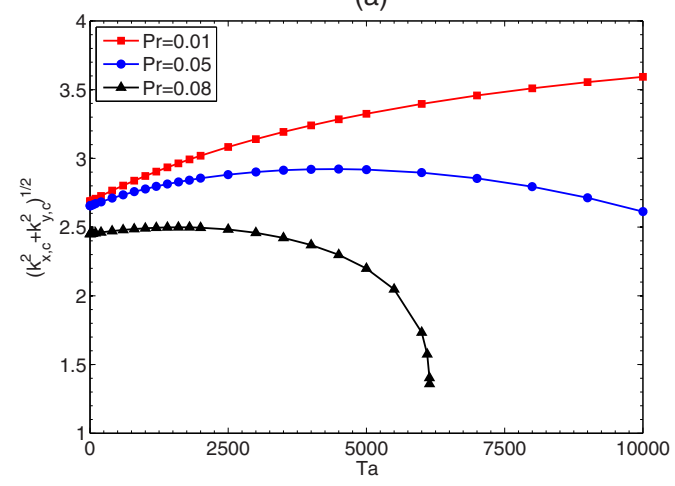

(b)

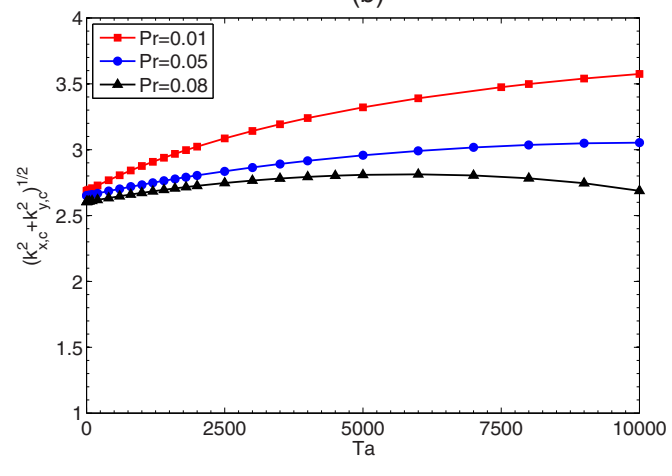

FIG. 9. Variation of the wave-number norm $\left|\vec{k}_{c}\right|$ for the stationary shear instability as a function of the Taylor number Ta for different Prandtl numbers Pr for (a) thermally insulating boundaries and (b) thermally conducting boundaries. 


\section{EFFECT OF ROTATION ON THE STABILITY OF SIDE- . . .}

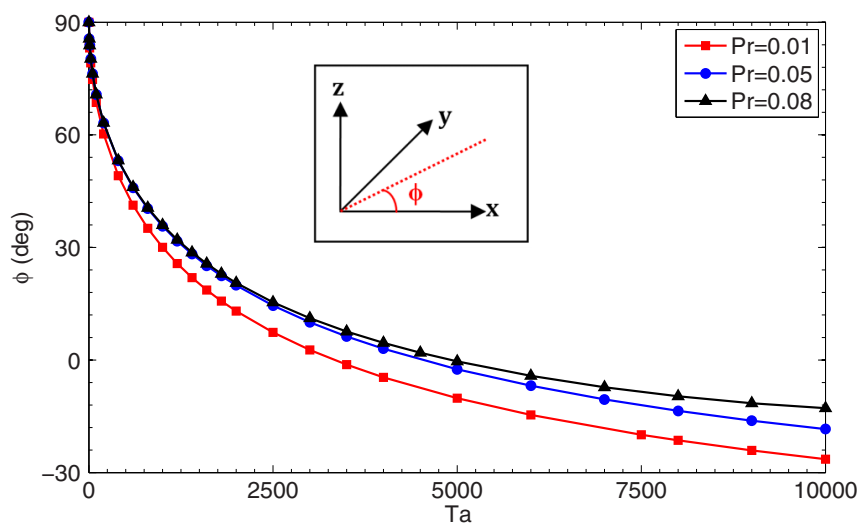

FIG. 10. Variation of $\phi$ (the roll axis angle in the $x y$ plane) for the stationary shear instability as a function of the Taylor number Ta for different Prandtl numbers $\operatorname{Pr}$ (thermally conducting boundaries).

is defined by only the component $k_{x, c}$ and $\phi=90^{\circ}$. When the Taylor number is increased, $\vec{k}_{c}$ starts to spin in the clockwise direction, thus creating a negative transverse component $k_{y, c}$. Its norm $\left|\vec{k}_{c}\right|$ undergoes a growth phase for the smallest values of Pr. This growth phase is shorter and weaker and is followed by a decrease phase for larger values of Pr, this decrease becoming very steep in the conducting case for $\operatorname{Pr}=0.08$.

The spinning of the wave vector in the clockwise direction is well depicted in Fig. 10 where the variation with Ta of $\phi$, the angle of the rolls axis with respect to the $x$ axis, is plotted. In this figure we show only the case of the thermally conducting walls since the observed variation in the case of insulating walls is typically the same. The angle $\phi$ evolves very quickly from $\phi=90^{\circ}$ for $\mathrm{Ta}=0$ to $\phi=0^{\circ}$ for moderate values of Ta depending on $\operatorname{Pr}(\mathrm{Ta} \approx 3250$ for $\operatorname{Pr}=0.08$, Ta $\approx 4500$ for $\operatorname{Pr}=0.05$, and $\mathrm{Ta} \approx 5000$ for $\operatorname{Pr}=0.01$ ), indicating a change of the shear modes from transverse to longitudinal. The variation of $\phi$ is very steep at small Ta, in a domain where the basic flow also strongly changes its orientation in the clockwise direction due to the creation of the transverse velocity component as a result of the applied rotation. The spinning of the wave vector continues more slowly beyond the longitudinal orientation of the rolls with negative values of $\phi$. Note that the decrease of $\phi$ with Ta does not depend much on Pr: It is only slightly less steep when Pr is increased. Such a deviation of the wave vector when the Taylor number is increased was already mentioned by Chandrasekhar [22] in the case of rotating Rayleigh-Bénard convection. An illustration of the change of the marginal eigenvector with the applied rotation is given in Fig. 11: The modification
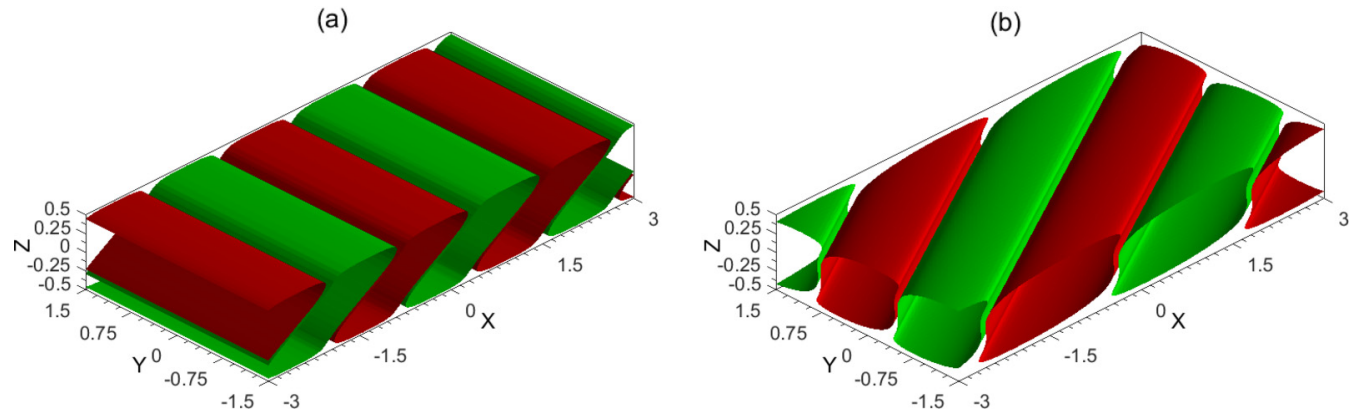

FIG. 11. Structure of the eigenvector $(\mathrm{a})$ without $(\mathrm{at} \mathrm{Ta}=0)$ and $(\mathrm{b})$ with $($ at $\mathrm{Ta}=1000)$ rotation for the stationary shear instability at the marginal state $(\operatorname{Pr}=0.01$, thermally conducting boundaries). The isosurfaces correspond to [plus (green) or minus (red)] a given value of the temperature perturbation. 
(a)

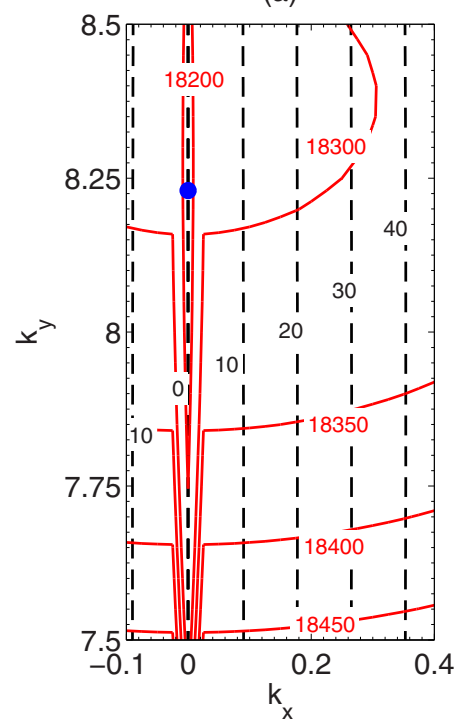

(b)

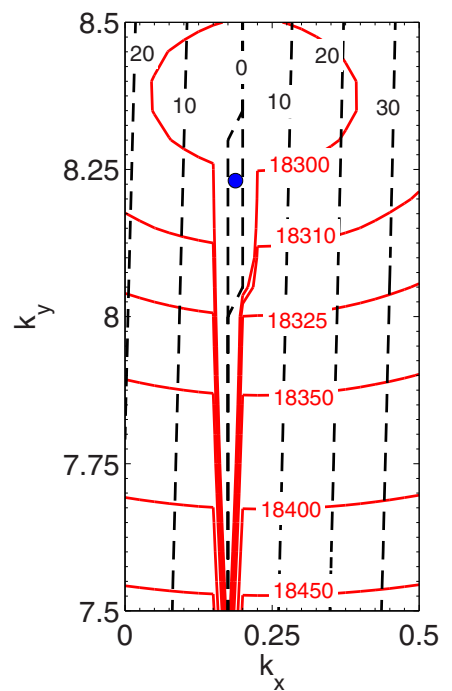

(c)

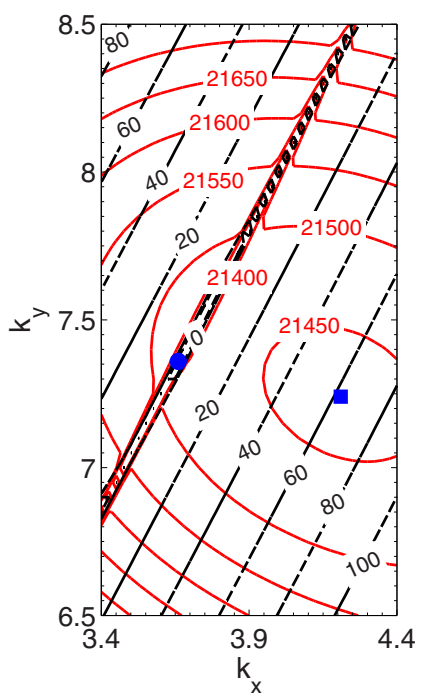

FIG. 12. Isovalues of the thresholds $\mathrm{Gr}_{0}$ for the Rayleigh instability (red solid lines) and of the corresponding angular frequency $\omega$ (black dashed lines) in the wave-number plane $\left(k_{x}, k_{y}\right)$ for $\operatorname{Pr}=0.7$ and (a) $\mathrm{Ta}=0$, (b) $\mathrm{Ta}=1$, and (c) $\mathrm{Ta}=500$. The blue points indicate the position in the $\left(k_{x}, k_{y}\right)$ space of the critical stationary thresholds (circles) and, in (c), of the oscillatory threshold (square).

of the marginal roll orientation is well depicted. The marginal roll structure has also changed: The inclined rolls obtained for $\mathrm{Ta}=0$ become rather square rolls at $\mathrm{Ta}=1000$, in connection with the decreasing velocity in the basic flow when rotation is applied.

\section{B. Thermal (Rayleigh) instabilities}

In the case of the thermal instabilities occurring for thermally conducting boundaries, the study was performed for two values of the Prandtl number: $\operatorname{Pr}=0.7$ and 7 . These two values correspond to typical values for air and water, respectively. Gershuni et al. [11] reported the existence of two modes for this instability: a stationary longitudinal mode and an oscillatory transverse mode. They showed that the longitudinal mode is the most unstable. In this work, for different cases, we have determined the two thresholds $\mathrm{Gr}_{c}^{L}$ (stationary longitudinal mode) and $\mathrm{Gr}_{c}^{T}$ (oscillatory transverse mode) and we always found that $\mathrm{Gr}_{c}^{T}>\mathrm{Gr}_{c}^{L}$, which is consistent with the paper of Gershuni et al. [11].

We first draw attention to the fact that the critical threshold for this instability is very difficult to locate when the rotation is introduced. The main reason is that the stationary mode (with smaller threshold for $\mathrm{Ta}=0$ ) is localized in a very thin region of the wave-number space, which is itself inside a large area where the mode is oscillatory. For $\mathrm{Ta}=0$, the stationary mode is longitudinal and can be found quite easily by setting $k_{x}$ equal to 0 . In contrast, under the influence of the rotation, the stationary mode becomes oblique and has to be found in the $\left(k_{x}, k_{y}\right)$ space, which becomes more and more difficult as Ta is increased. Indeed, the minimization procedure will more easily find the minimum for the oscillatory mode, which has a large basin of existence in the $\left(k_{x}, k_{y}\right)$ space.

The situation will be more clearly depicted in Fig. 12, where the thresholds for these instabilities, as well as the corresponding angular frequencies, are given in the wave-number plane $\left(k_{x}, k_{y}\right)$ for $\mathrm{Pr}=0.7$ and three values of the Taylor number, Ta $=0$ [Fig. 12(a)], Ta $=1$ [Fig. 12(b)], and $\mathrm{Ta}=500$ [Fig. 12(c)]. We can see the very localized region for the stationary mode corresponding to $\omega=0$. This region, originally elongated along the $k_{y}$ axis for $\mathrm{Ta}=0$, is, at the same time, moved to positive $k_{x}$ and inclined to the right when the rotation is applied. We can also see the large basin 

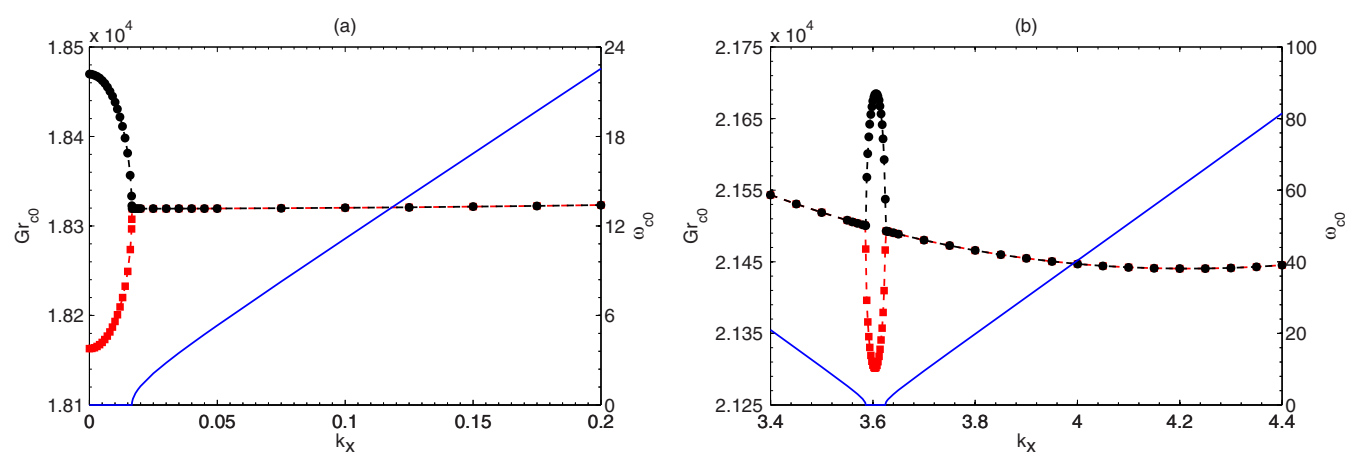

FIG. 13. Thresholds $\mathrm{Gr}_{0}$ for the Rayleigh instability (dashed lines) and corresponding angular frequency (solid lines) as a function of the $k_{x}$ wave number for $\operatorname{Pr}=0.7$. The thresholds are given for both the first (dashed lines with squares) and second (dashed lines with circles) unstable eigenvalues at (a) $k_{y}=8$ for Ta $=0$ and (b) $k_{y}=7.25$ for $\mathrm{Ta}=500$.

of existence for the oscillatory mode. Originally symmetric with respect to the $k_{y}$ axis at $k_{x}=0$ for $\mathrm{Ta}=0$, this basin is also moved towards positive $k_{x}$ and tilted to the right.

In order to better understand how the stationary and oscillatory modes are connected, the thresholds and angular frequencies are now plotted as a function of $k_{x}$ at constant $k_{y}$, close to the critical values, in Fig. 13. The thresholds corresponding to the two leading eigenvalues are plotted at $\mathrm{Pr}=0.7$ for Ta $=0$ [Fig. 13(a)] and Ta $=500$ [Fig. 13(b)]. For the two values of Ta, we see that the critical stationary mode is created locally from the oscillatory mode. More precisely, the two complex-conjugate eigenvalues associated with the complex-conjugate oscillatory modes collide on the real axis (their angular frequency goes to zero) and then split, in a very narrow $k_{x}$ interval, into two real eigenvalues associated with two stationary modes, one of these modes being the critical mode (conversely, we also characterize that as a collision of the steady eigenvalues). The stationary and oscillatory Rayleigh modes are then in close connection, one being in continuity with the other.

Figures 12 and 13 can also give indications of the different thresholds and the difficulties to get them. For Ta $=0$ [Figs. 12(a) and 13(a)], the $k_{x}$ interval for the stationary mode is at the minimum of the oscillatory thresholds in the wave-number domain. For $\mathrm{Ta}=0$ (and also for weak values of Ta), the basin of attraction of the oscillatory mode will then naturally lead the minimization to find the critical stationary threshold. In contrast, for large values of $\mathrm{Ta}$ as $\mathrm{Ta}=500$ [Figs. 12(c) and $13(\mathrm{~b})$ ], the minimums of the oscillatory and stationary thresholds have different positions in the $\left(k_{x}, k_{y}\right)$ space. Due to its large basin of attraction, the critical oscillatory threshold will be found easily by the minimization; however, strategies are needed to find the critical stationary threshold. We have used the fact that the critical stationary threshold is not very far in the $\left(k_{x}, k_{y}\right)$ space from the critical oscillatory threshold: We calculate the instability threshold in the $\left(k_{x}, k_{y}\right)$ domain close to the critical oscillatory threshold until the localization of the stationary instability and we then apply the minimization procedure. Note finally that the different localizations of the minimums for the stationary and oscillatory thresholds in the $\left(k_{x}, k_{y}\right)$ space (as observed for Ta $=500$ ) could allow the oscillatory instability to become eventually dominant when Ta is further increased.

Figure 14 depicts the dependence of the critical Grashof numbers $\mathrm{Gr}_{c}$ for the Rayleigh instability on the Taylor number Ta. The thresholds for both stationary and oscillatory instabilities are given for $\operatorname{Pr}=0.7$ [Fig. 14(a)] and $\mathrm{Pr}=7$ [Fig. 14(b)]. We see that, in the range of Ta studied, $\mathrm{Gr}_{c}$ grows almost linearly with respect to the Taylor number in all cases. This indicates a stabilization of the basic flow due to the rotation. In addition, the thresholds for the stationary and oscillatory instabilities remain very close to each other. For $\operatorname{Pr}=0.7$ [Fig. 14(a)], however, it is observed that the stationary mode remains the dominant mode in the range of Ta studied. In contrast, for $\operatorname{Pr}=7$ [Fig. 14(b)], 
(a)

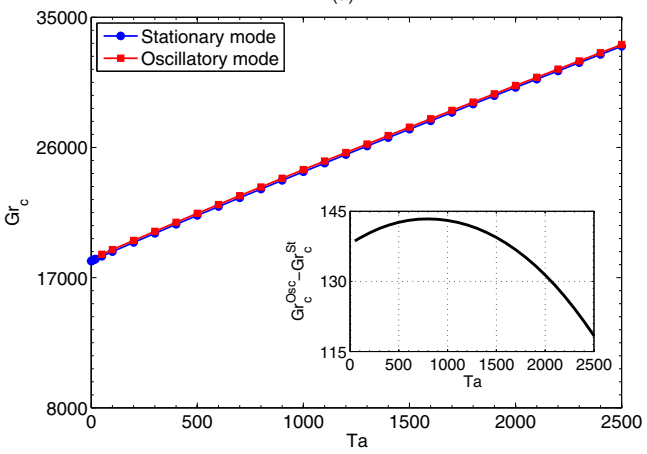

(b)

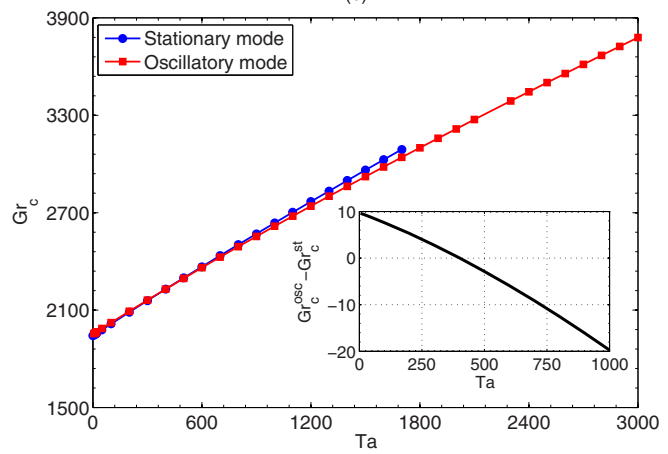

FIG. 14. Critical Grashof numbers $\mathrm{Gr}_{c}$ for the Rayleigh instabilities as a function of the Taylor number Ta for (a) $\operatorname{Pr}=0.7$ and (b) $\operatorname{Pr}=7$. The thresholds for both stationary and oscillatory modes are presented. The insets give $\mathrm{Gr}_{c}^{\text {osc }}-\mathrm{Gr}_{c}^{\text {st }}$ to highlight the possible crossing of the critical curves.

the stationary mode is dominant only for Ta up to approximately 400. Beyond this value, due to a crossing of the critical curves, the oscillatory mode becomes dominant. Note that, for $\operatorname{Pr}=0.7$, extrapolation of both critical curves to larger Ta also indicates a possible crossing of the curves for $\mathrm{Ta} \approx 4400$.

The norms of the critical wave number associated with these instabilities, as well as the critical angular frequency for the oscillatory mode obtained at $\operatorname{Pr}=7$, are given in Figs. 15(a) and 15(b), respectively. For $\operatorname{Pr}=0.7$, the wave number slightly decreases, reaches a minimum at $\mathrm{Ta} \approx 300$, and begins to grow, quickly reaching a linear law. In contrast, for $\operatorname{Pr}=7$, the wave numbers corresponding to both stationary and oscillatory instabilities grow linearly with respect to the Taylor number and the angular frequency grows as a power law of the type $C \mathrm{Ta}^{n}$ with $C=1.33$ and $n=0.47$.

The deviation of the critical rolls when the Taylor number Ta is increased is depicted in Fig. 16. For $\mathrm{Ta}=0$, the modes are longitudinal and thus the angle $\phi$ between the rolls axis and the $x$ axis is equal to zero. When Ta is increased, the modes become oblique modes, with a deviation in the clockwise direction. The deviation is weaker than for the transverse modes of the shear instability. It appears to be the same for $\operatorname{Pr}=0.7$ and 7 in the case of the stationary instability and to be more accentuated for the oscillatory instability. An illustration of the marginal roll orientation change when rotation is applied is given in Fig. 17. Note also that, in both cases (without or with rotation),

(a)

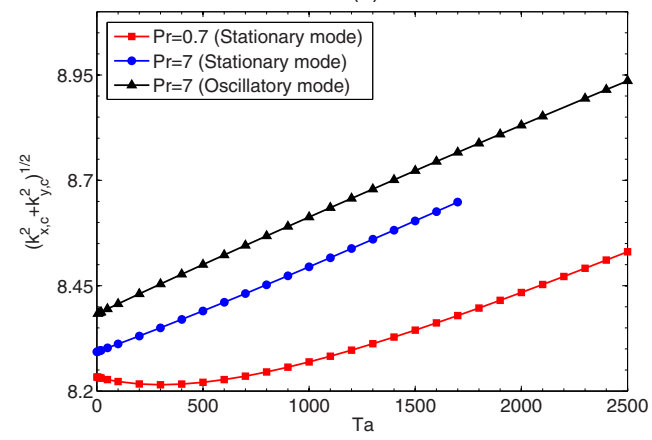

(b)

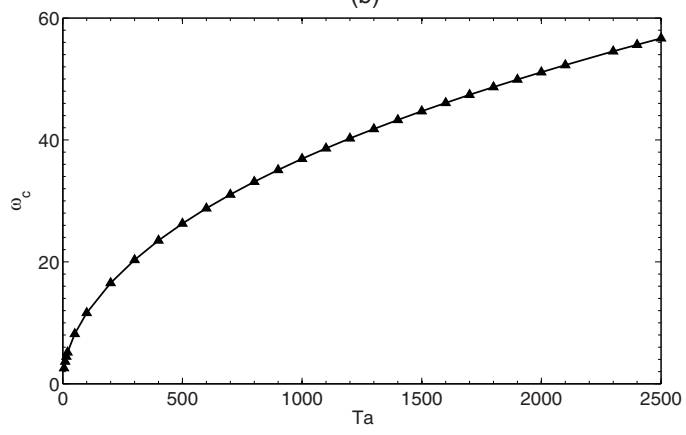

FIG. 15. Critical properties of the Rayleigh instabilities as a function of the Taylor number Ta for different Pr numbers: (a) wave-number norm $\left|\vec{k}_{c}\right|$ and (b) angular frequency $\omega_{c}$ of the oscillatory mode at $\operatorname{Pr}=7$. 


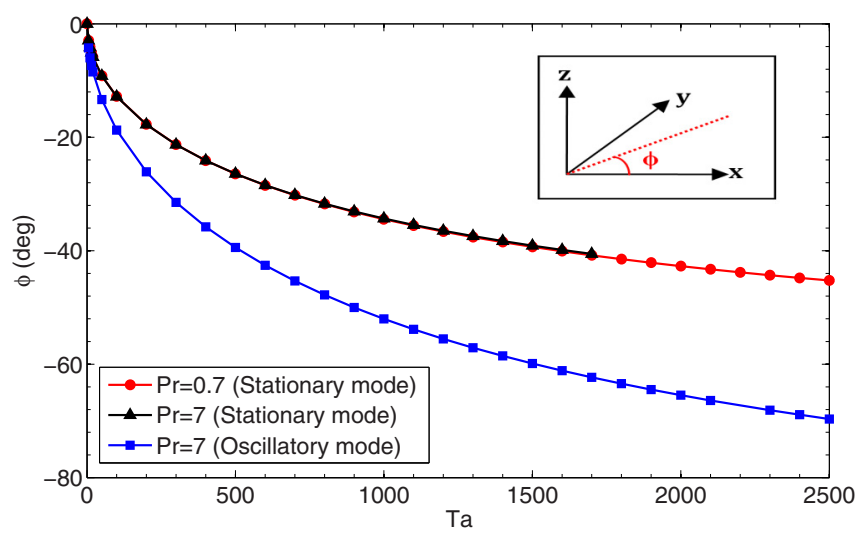

FIG. 16. Variation of $\phi$ (the roll axis angle in the $x y$ plane) for the Rayleigh instability as a function of the Taylor number Ta for different Prandtl numbers Pr.

the marginal rolls are confined in the upper or lower part of the cavity, i.e., in both zones where the basic temperature gradient is destabilizing.

\section{Oscillatory instability}

The influence of rotation on the oscillatory instability was studied for both thermal boundary conditions and in each case for different values of the Prandtl number. The stability curves showing the critical thresholds as a function of the Taylor number Ta are given in Fig. 18. For both boundary conditions, we observe a stabilization of the oscillatory instability when rotation is applied. For insulating walls [Fig. 18(a)], two regimes are found as a function of the Taylor number: a first regime at small Ta where the stabilization is moderate and the thresholds evolve almost linearly with Ta and another regime for larger Ta with a steep increase of the thresholds. The increase of the Prandtl number induces a sooner transition between the regimes (at $\mathrm{Ta} \approx 7500$ for $\mathrm{Pr}=0.05, \mathrm{Ta} \approx 3000$ for $\operatorname{Pr}=0.1$, and $\mathrm{Ta} \approx 1000$ for $\operatorname{Pr}=0.15$ ) and a steeper increase of the thresholds. Conversely, for conducting walls [Fig. 18(b)], the stabilization of the flow is more regular and the same evolution of the thresholds $\mathrm{Gr}_{c}$ with Ta is found for the different values of the Prandtl number. For the smallest $\operatorname{Pr}$ values $(\operatorname{Pr}=0.1$ and 0.15$)$, however, a smaller slope of the stability curves $\mathrm{Gr}_{c}(\mathrm{Ta})$ is found for values of Ta above 6000 .
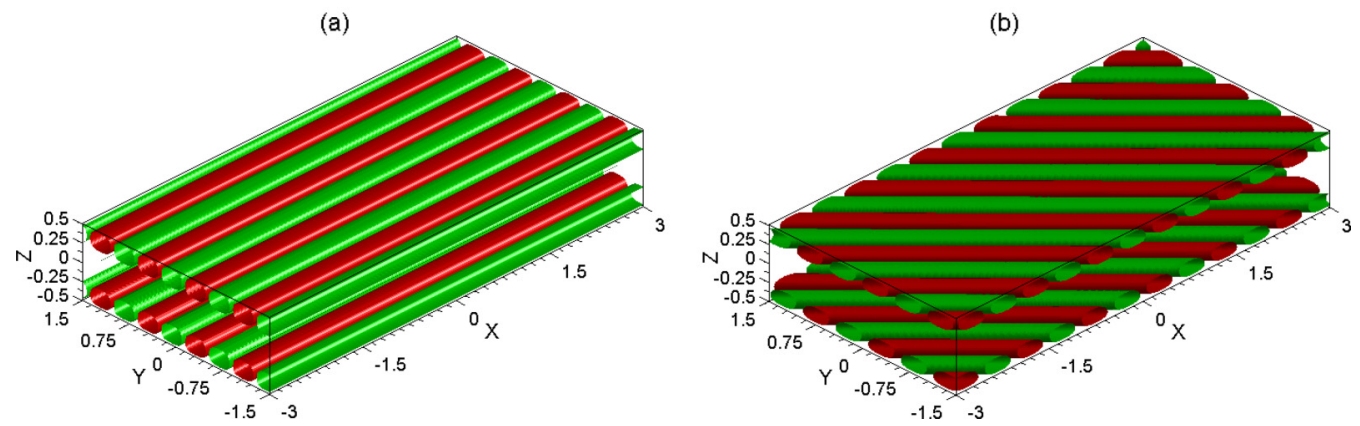

FIG. 17. Structure of the eigenvector (a) without (at $\mathrm{Ta}=0$ ) and (b) with (at $\mathrm{Ta}=2500)$ rotation for the stationary Rayleigh instability at the marginal state $(\mathrm{Pr}=0.7$ and thermally conducting boundaries). The isosurfaces correspond to [plus (green) or minus (red)] a given value of the temperature perturbation. 
(a)

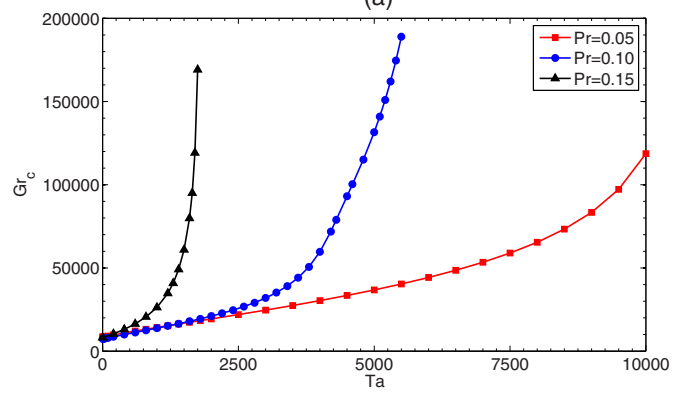

(b)

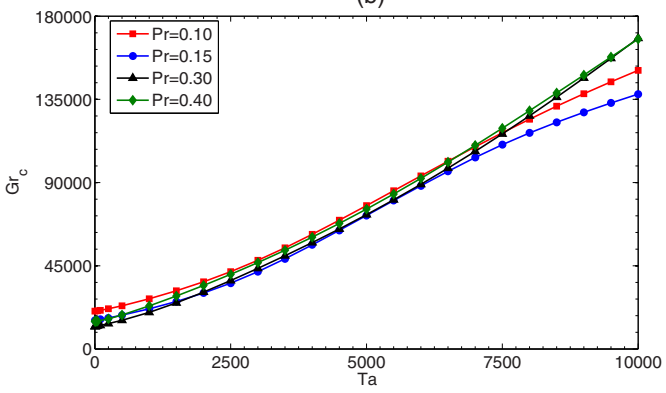

FIG. 18. Variation of the thresholds $\mathrm{Gr}_{c}$ for the oscillatory instability as a function of the Taylor number Ta for different Prandtl numbers Pr for (a) thermally insulating boundaries and (b) thermally conducting boundaries.

The change of the wave-number norm with Ta for the oscillatory instability is shown in Fig. 19. For the insulating boundaries [Fig. 19(a)], the critical wave number $\left|\vec{k}_{c}\right|$ increases for small Taylor numbers, reaches a maximum, and finally decreases. This behavior depends on the Prandtl number: When Pr is increased, the maximum decreases, occurs for smaller Ta, and the final decrease is steeper. For $\operatorname{Pr}=0.1$, we can also note a kink occurring during the final decrease. The behavior is quite different for conducting boundaries [Fig. 19(b)]. For $\operatorname{Pr}=0.3$ and 0.4, the critical wave number still experiences an initial increase and then a decrease when Ta is increased, but these changes remain moderate. In contrast, for $\operatorname{Pr}=0.1$ and 0.15 , after a slow variation of the critical wave number with Ta, we observe a drift of the wave-number norm to larger values. This indicates that, for these Pr values, the smaller thresholds observed in Fig. 18(b) when Ta is increased have been found for large wave numbers.

The orientation of the oscillatory mode under the influence of the rotation is depicted in Fig. 20. For $\mathrm{Ta}=0$, the critical mode is longitudinal, i.e., it corresponds to $\phi=0$. For insulating boundaries [Fig. 20(a)], the rolls are globally deviated in the clockwise direction, roughly following the same curve for the different $\operatorname{Pr}$ values. This clockwise deviation, however, is limited up to some value of Ta depending on the Prandtl number $(\mathrm{Ta} \approx 3750$ for $\operatorname{Pr}=0.1$, and $\mathrm{Ta} \approx 750$ for $\mathrm{Pr}=0.15$ ) and a slighter counterclockwise deviation can then be observed. The largest clockwise deviations are thus observed for the smaller values of Pr. For conducting boundaries [Fig. 20(b)], the deviation evolves regularly in the clockwise direction. It is the weakest for $\operatorname{Pr}=0.4$ and 0.3 and the largest for

(a)

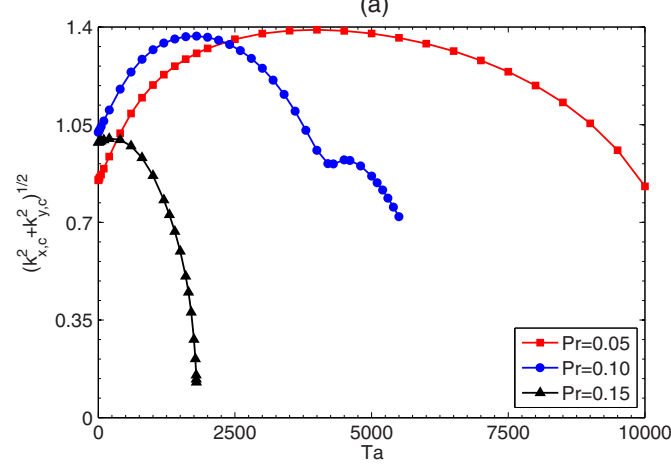

(b)

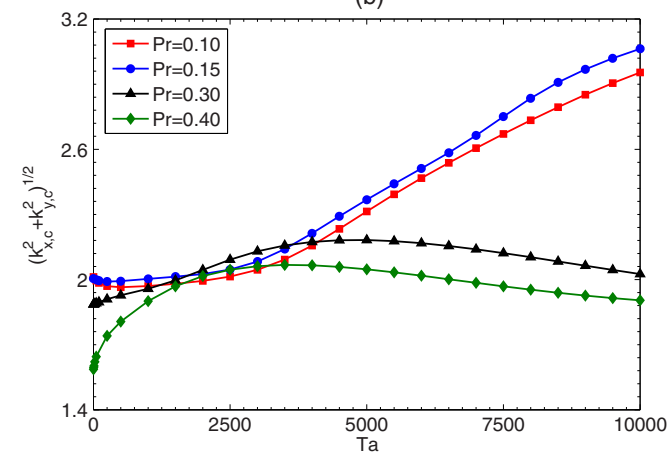

FIG. 19. Variation of the wave-number norm $\left|\vec{k}_{c}\right|$ for the oscillatory instability as a function of the Taylor number Ta for different Prandtl numbers Pr for (a) thermally insulating boundaries and (b) thermally conducting boundaries. 
(a)

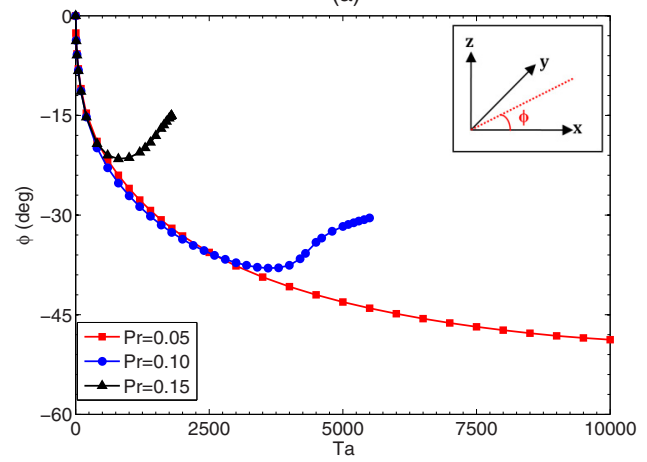

(b)

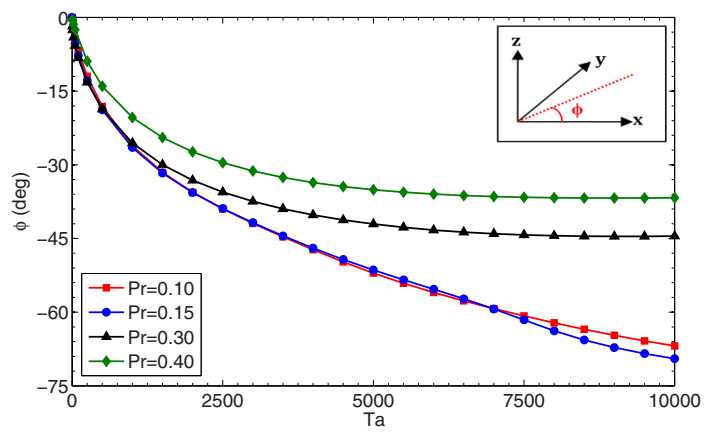

FIG. 20. Variation of $\phi$ (the roll axis angle in the $x y$ plane) for the oscillatory instability as a function of the Taylor number Ta for different Prandtl numbers Pr for (a) thermally insulating boundaries and (b) thermally conducting boundaries.

$\operatorname{Pr}=0.15$ and 0.1 . For these latter values of $\mathrm{Pr}$, this largest deviation associated with the strongest increase of the wave-number norm [Fig. 19(b)] indicates the strong increase with Ta of a positive $k_{x, c}$ component.

Concerning the critical angular frequency $\omega_{c}$ for these oscillatory modes (Fig. 21), it increases under the influence of the rotation and the increase is stronger when $\operatorname{Pr}$ is increased. For insulating boundaries [Fig. 21(a)], we still observe two regimes: the first regime with a moderate increase of $\omega_{c}$ and the second regime with a steeper increase. Steeper regimes are found when Pr is increased and the change of regime occurs earlier. For conducting boundaries [Fig. 21(b)], the critical frequency evolves more regularly, but with a globally more important increase. Note that for $\operatorname{Pr}=0.4$ the increase of $\omega_{c}$ with Ta is almost linear.

\section{ENERGY ANALYSIS}

Important information concerning the physical mechanisms involved in the transition to the instability and in the stabilization by the applied rotation can be obtained from the calculation, at threshold, of the fluctuating kinetic energy budget. The basic steady solution at threshold and the critical eigenvector both enter the equation of energy budget. Here we recall some mathematical definitions of these budgets, which we will present and discuss in the next section.
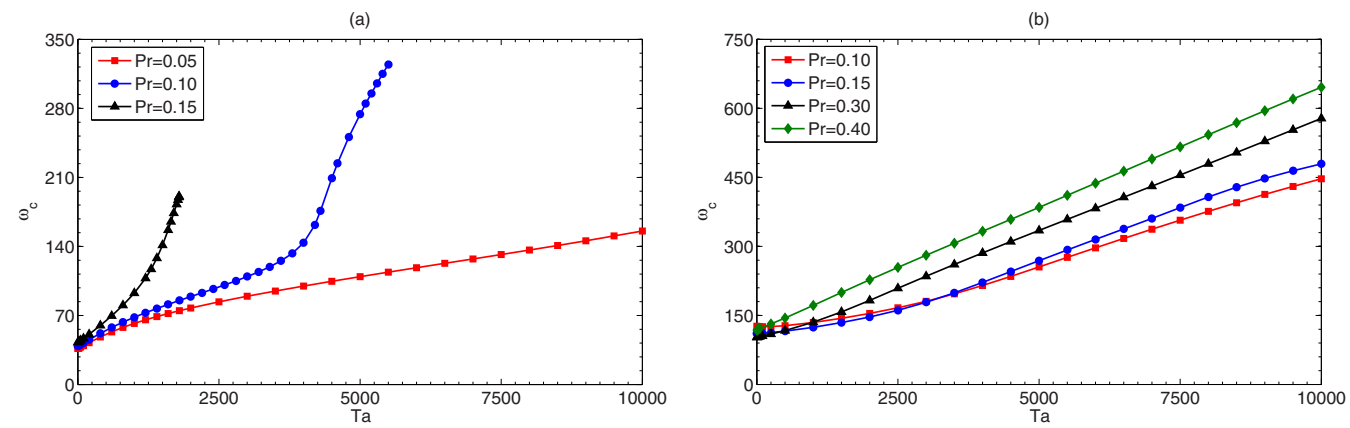

FIG. 21. Variation of the critical angular frequency $\omega_{c}$ for the oscillatory instability as a function of the Taylor number Ta for different Prandtl numbers Pr for (a) thermally insulating boundaries and (b) thermally conducting boundaries. 


\section{A. Fluctuating kinetic energy}

The fluctuating kinetic energy is defined by the scalar product of the disturbance by its complex conjugate $k=\operatorname{Re}\left(u_{i} u_{i}^{*} / 2\right.$ ) (Re and the asterisk superscript denoting the real part and the complex conjugate, respectively). The equation governing the budget of the kinetic energy is given by

$$
\frac{\partial k}{\partial t}=k_{s, U}+k_{s, V}+k_{\text {pres }}+k_{\text {buoy }}+k_{\text {visc }},
$$

where $\partial k / \partial t$ is the rate of change of the fluctuating kinetic energy, $k_{s, U}=-\operatorname{Re}\left(w u^{*} \frac{d U_{b}}{d z}\right)$ and $k_{s, V}=-\operatorname{Re}\left(w v^{*} \frac{d V_{b}}{d z}\right)$ represent the production of fluctuating kinetic energy by shear of the basic flow, $k_{\text {pres }}=-\operatorname{Re}\left(u^{*} \frac{\partial p}{\partial x}+v^{*} \frac{\partial p}{\partial y}+w^{*} \frac{\partial p}{\partial z}\right)$ is the redistribution of fluctuating kinetic energy by the pressure fluctuations, $k_{\text {buoy }}=\operatorname{Re}\left(\mathrm{Gr} w^{*} \theta\right)$ is the buoyancy contribution to the fluctuating kinetic energy, and finally $k_{\mathrm{visc}}=\operatorname{Re}\left(u^{*} \nabla^{2} u+v^{*} \nabla^{2} v+w^{*} \nabla^{2} w\right)$ is the viscous dissipation of fluctuating kinetic energy. Note that the two terms connected with the rotation do not contribute to the kinetic energy budget [Eq. (23)] as they have opposite contributions that are annihilated. The contribution of the rotation will then only be viewed through the change of the basic flow quantities and perturbations.

We can also define the total fluctuating kinetic energy as $K=\int_{V} k d V$. The rate of change of $K$ is thus given by the volume integral energy terms (denoted by $K_{i}$ )

$$
\frac{d K}{d t}=K_{s, U}+K_{s, V}+K_{\text {buoy }}+K_{\text {visc }} .
$$

The volume integral pressure term is zero and therefore has not been included in Eq. (24). Note that the disturbances associated with the critical eigenvector are defined to within a multiplicative constant and need to be appropriately normalized. This normalization will be done with the viscous dissipation, more precisely $\left|K_{\text {visc }}\right|=-K_{\text {visc }}$, using the fact that $K_{\text {visc }}$ is stabilizing by nature and thus a negative term $\left[K_{\text {visc }}=\int_{V}\left(\vec{v} \cdot \nabla^{2} \vec{v}\right) d V=-\int_{V}(\vec{\nabla} \vec{v})^{2} d V\right]$. Moreover, at threshold, the critical eigenvector is associated with an eigenvalue of zero real part. This implies that $\partial k / \partial t$ and $\partial K / \partial t$ are both equal to zero at marginal stability.

Applying the normalization to Eq. (24), we get a new equation involving normalized energy terms at threshold $K_{i}^{\prime}=K_{i} /\left|K_{\text {visc }}\right|$ :

$$
K_{s, U}^{\prime}+K_{s, V}^{\prime}+K_{\text {buoy }}^{\prime}=1 .
$$

Conversely, each energy term in Eq. (23) can be normalized by $\left|K_{\text {visc }}\right|$, which yields $k_{i}^{\prime}$ terms and, at the threshold, we get

$$
k_{s, U}^{\prime}+k_{s, V}^{\prime}+k_{\text {pres }}^{\prime}+k_{\text {buoy }}^{\prime}+k_{\text {visc }}^{\prime}=0 .
$$

Note that all terms $K_{i}^{\prime}$ and $k_{i}^{\prime}$ have been normalized and are then intrinsic quantities of the flow perturbations. The calculation of all the individual energy contributions $K_{i}^{\prime}$ enables us to determine which term plays a dominant role in triggering the instability through production of fluctuating kinetic energy. The corresponding spatial fields $k_{i}^{\prime}$ can in turn be analyzed to locate the production regions. Positive (negative) energy terms are destabilizing (stabilizing).

\section{B. Fluctuating thermal energy}

The thermal energy balance could also be of interest, particularly in the case of the thermal Rayleigh instability. Hence, by taking the equation of the temperature fluctuation [Eq. (20)], multiplying it by the complex conjugate of the temperature disturbance, and isolating the real part, we obtain an equation related to the production rate of the fluctuating thermal energy $e=\operatorname{Re}\left(\theta \theta^{*} / 2\right)$ :

$$
\frac{\partial e}{\partial t}=e_{l}+e_{v}+e_{d}
$$


where $e_{l}=-\operatorname{Re}\left(\theta^{*} u \frac{\partial \Theta_{b}}{\partial x}\right)=-\operatorname{Re}\left(\theta^{*} u\right)$ represents a production term related to the imposed longitudinal gradient of temperature, $e_{v}=-\operatorname{Re}\left(\theta^{*} w \frac{\partial \Theta_{b}}{\partial z}\right)=-\operatorname{Re}\left(\theta^{*} w \frac{d T_{b}}{d z}\right)$ represents a production term related to the vertical gradient of temperature, and $e_{d}=\operatorname{Pr} \operatorname{Re}\left[\theta^{*}\left(\frac{d^{2} \theta}{d z^{2}}-k^{2} \theta\right)\right]$ is a dissipation term linked to heat diffusion. Similarly to (27), we can also obtain an equation for the total fluctuating thermal energy $E=\int_{V} e d v$ :

$$
\frac{\partial E}{\partial t}=E_{l}+E_{v}+E_{d}
$$

where $E_{l}, E_{v}$, and $E_{d}$ are the volume integral quantities related to the local distributions $e_{l}, e_{v}$, and $e_{d}$, respectively. After normalization with respect to $\left|E_{d}\right|=-E_{d}$ and at the threshold, we get

$$
e_{l}^{\prime}+e_{v}^{\prime}+e_{d}^{\prime}=0
$$

and

$$
E_{l}^{\prime}+E_{v}^{\prime}=1
$$

\section{Contributions to the critical Grashof number}

It is also interesting to see through which energetic contributions the rotation will act on the critical thresholds. The approach we propose is to express the critical Grashof number, given in relation to the reference case without rotation, as a function of different energetic contributions expressed as characteristic ratios. To do this, we recall that the basic flow $\left[U_{b}(z), V_{b}(z)\right]$ is directly proportional to $\mathrm{Gr}, U_{b}(z)=\mathrm{Gr} u_{b}(z)$, and $V_{b}(z)=\mathrm{Gr} v_{b}(z)$. Thus, Gr can be factored out of the shear energy terms, so $K_{s, U}^{\prime}=\operatorname{Gr} K_{s, U}^{\prime \prime}$ and $K_{s, V}^{\prime}=\operatorname{Gr} K_{s, V}^{\prime \prime}$. A similar transformation applies to the energy due to buoyancy, which can be written as $K_{\text {buoy }}^{\prime}=\operatorname{Gr} K_{\text {buoy }}^{\prime \prime}$. Equation (25) at the marginal stability can then be written as

$$
\operatorname{Gr}_{c}\left(K_{s, U}^{\prime \prime}+K_{s, V}^{\prime \prime}+K_{\text {buoy }}^{\prime \prime}\right)=1 .
$$

Taking account of the fact that $K_{s, V}^{\prime \prime}=0$ in the case without rotation $(\mathrm{Ta}=0)$, Eq. (31) can be used to write

$$
\frac{\mathrm{Gr}_{c 0}}{\mathrm{Gr}_{c}}=\overbrace{\left(\frac{K_{s, U}^{\prime \prime}}{K_{s, U 0}^{\prime \prime}+K_{\text {buoy } 0}^{\prime \prime}}\right)}^{R_{U}^{K}}+\overbrace{\left(\frac{K_{s, V}^{\prime \prime}}{K_{s, U 0}^{\prime \prime}+K_{\text {buoy } 0}^{\prime \prime}}\right)}^{R_{V}^{K}}+\overbrace{\left(\frac{K_{\text {buoy }}^{\prime \prime}}{K_{s, U 0}^{\prime \prime}+K_{\text {buoy } 0}^{\prime \prime}}\right)}^{R_{b}^{K}} .
$$

Concerning (30), we can also factorize GrPr from $E_{v}^{\prime}$, which yields $E_{v}^{\prime}=\operatorname{Gr} \operatorname{Pr} E_{v}^{\prime \prime}$. By introducing this in Eq. (30), after some simple manipulations we obtain

$$
\frac{\mathrm{Gr}_{c 0}}{\mathrm{Gr}_{c}}=\overbrace{\left(\frac{1-E_{l 0}^{\prime}}{1-E_{l}^{\prime}}\right)}^{1 / R_{l}^{E}} \overbrace{\left(\frac{E_{v}^{\prime \prime}}{E_{v 0}^{\prime \prime}}\right)}^{R_{v}^{E}}=\frac{R_{v}^{E}}{R_{l}^{E}} .
$$

Note that the values with the subscript 0 refer to the case without rotation $(\mathrm{Ta}=0)$.

\section{ANALYSIS OF THE RESULTS}

In this section, the results obtained for the three types of instability are analyzed, using energy considerations when interesting. In the case of the shear instability $(\operatorname{Pr}=0.01)$ we choose to analyze the result corresponding to the case of the insulating walls. This choice is motivated by the fact that buoyancy does not play a significant role and thus the nature of the boundary condition (insulation or conduction) does not affect the results. Contrarily to this case, the oscillatory instability $(\operatorname{Pr}=0.1)$ is affected by the nature of the boundary condition and thus we will consider the two cases. The 
(a)

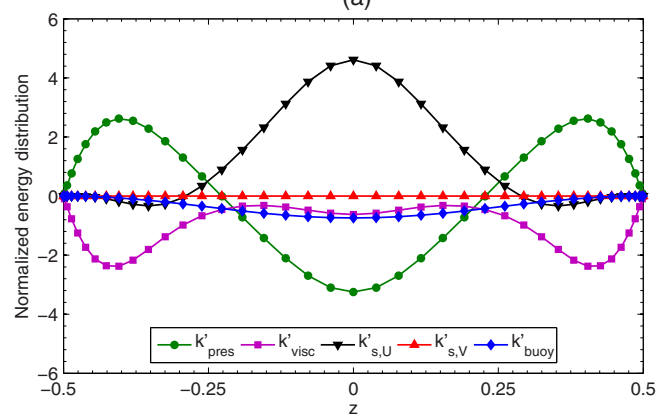

(c)

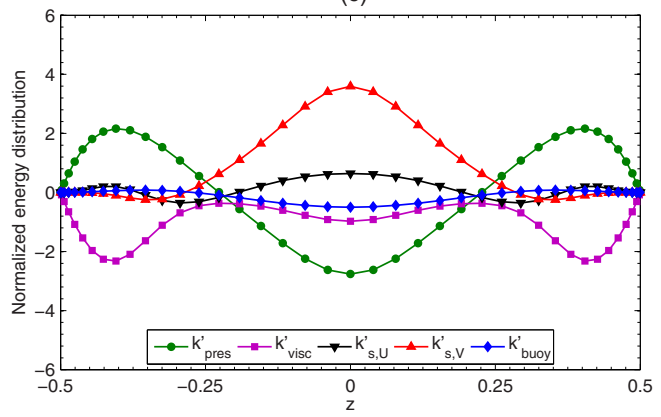

(b)

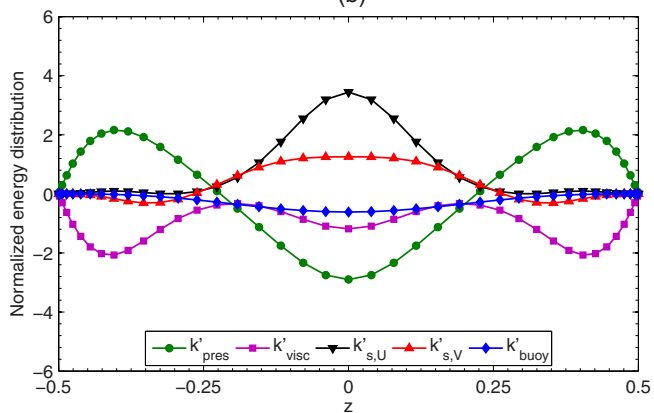

(d)

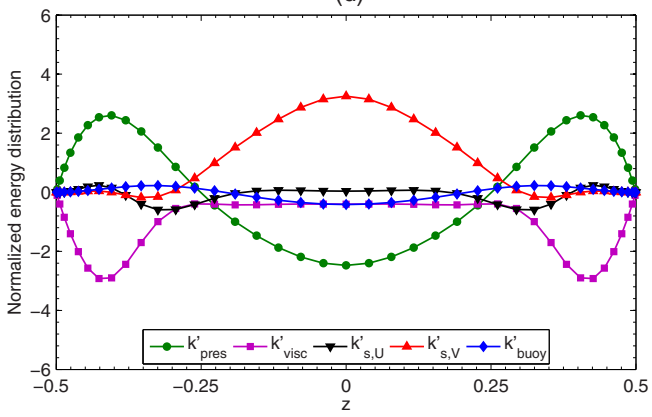

FIG. 22. Local kinetic energy contributions along $z$ for the shear instability at $\operatorname{Pr}=0.01$ and $(a)$ Ta $=0$, (b) $\mathrm{Ta}=1000$, (c) $\mathrm{Ta}=5000$, and (d) $\mathrm{Ta}=10000$.

Rayleigh instability is only present in the case of conductive walls, but we have noticed a significant change between the cases for $\operatorname{Pr}=0.7$ and $\operatorname{Pr}=7$. Hence, the two cases will be considered here.

\section{A. Shear instability}

The spatial fields $k_{i}^{\prime}$ corresponding to the different kinetic energy contributions for the shear instability are shown in Fig. 22 for different values of the Taylor number. For Ta $=0$ [Fig. 22(a)], we observe a strong destabilization associated with the longitudinal shear term $k_{s, U}^{\prime}$ in the center region $(z=0)$ where the inflection point of the basic profile is located, while the term $k_{s, V}^{\prime}$ is zero in the absence of rotation. A main stabilization contribution, principally located near the walls, is associated with the viscous term $k_{\text {visc }}^{\prime}$. The buoyancy term $k_{\text {buoy }}^{\prime}$ also acts as a negative contribution (stabilization), but it is very weak. Finally, the pressure term $k_{\text {pres }}^{\prime}$ ensures the local energetic equilibrium in the fluid layer by a transfer of energy from the production region at the center towards the dissipation zones along the walls. When the Taylor number is increased, the shear terms $k_{s, U}^{\prime}$ and $k_{s, V}^{\prime}$ are modified, particularly through the modification of the basic velocity profiles. The transverse shear energy term $k_{s, V}^{\prime}$ begins to grow while the term $k_{s, U}^{\prime}$ decreases, until $k_{s, V}^{\prime}$ becomes dominant [see Fig. 22(c) for $\mathrm{Ta}=5000]$. For larger values of $\mathrm{Ta}\left[\mathrm{Ta}=10^{4}\right.$; see Fig. 22(d)], these two contributions continue to evolve and reach a state in which the instability is triggered by the transverse velocity profile, while the longitudinal velocity contribution becomes zero at the center and even acts as a stabilization factor in the region between the walls and the center of the fluid layer.

The global kinetic energy contributions are given in Fig. 23(a) as a function of the Taylor number. We see that the buoyancy term, which remains weak and slightly negative, is negligible in the energy balance and that the main contributions to the destabilization come from the shear terms. For small Taylor numbers, the major contribution $K_{s, U}^{\prime}$ is related to the longitudinal shear term, which decreases as the Taylor number is increased. In contrast, the term $K_{s, V}^{\prime}$ related to the transverse velocity profile increases with Ta and becomes dominant for Ta $\approx 1800$. The decreasing $K_{s, U}^{\prime}$ term 

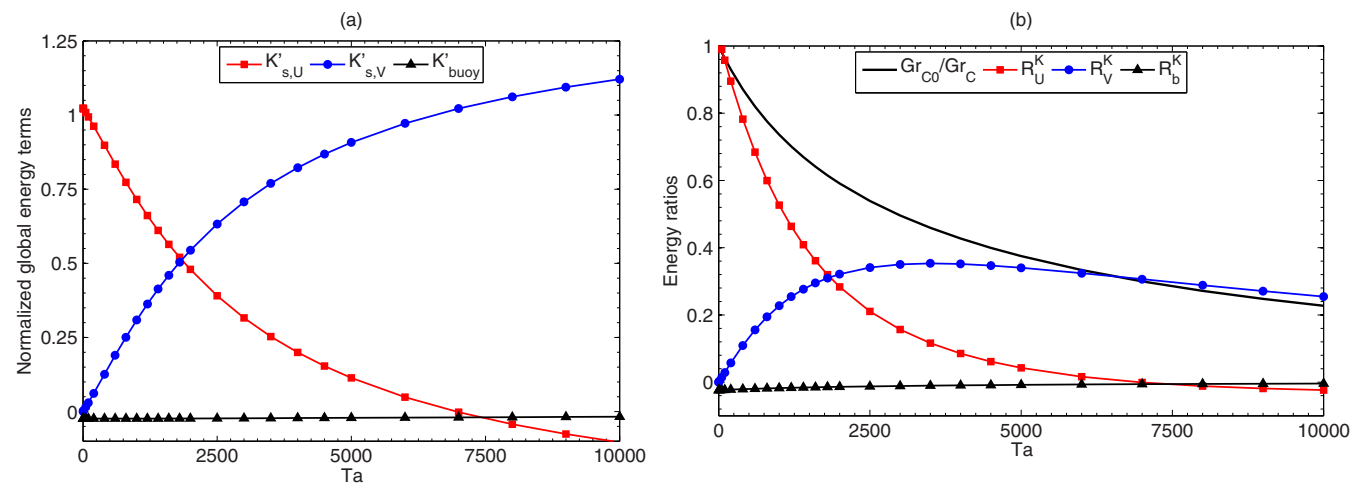

FIG. 23. (a) Contributions to the total fluctuating kinetic energy and (b) ratios $R_{U}^{K}, R_{V}^{K}$, and $R_{b}^{K}$ defining $\mathrm{Gr}_{c 0} / \mathrm{Gr}_{c}$ as a function of the Taylor number for the shear instability at $\mathrm{Pr}=0.01$.

eventually becomes negative for Ta $\approx 7000$, which means that, for larger Ta, only the $K_{s, V}^{\prime}$ term contributes to the destabilization.

To better show how these energy terms participate in the observed increase of the threshold $\mathrm{Gr}_{c}$ with Ta, Fig. 23(b) gives the ratios $R_{U}^{K}, R_{V}^{K}$, and $R_{b}^{K}$ defined in Eq. (32), as well as $\mathrm{Gr}_{c 0} / \mathrm{Gr}_{c}$, as a function of the Taylor number. Note first that the buoyancy contribution $R_{b}^{K}$ remains very small for these shear instabilities. Without rotation $(\mathrm{Ta}=0), R_{V}^{K}$ is equal to zero (no transverse flow) and $\mathrm{Gr}_{c 0} / \mathrm{Gr}_{c}=R_{U}^{K}+R_{b}^{K}=1 \approx R_{U}^{K}$. When Ta is increased, the stabilization effect corresponds to the decrease of the quantity $\mathrm{Gr}_{c 0} / \mathrm{Gr}_{c}$. This quantity is the sum of the rapidly decaying term $R_{U}^{K}$, which even becomes negative at Ta $\approx 7000$, and the term $R_{V}^{K}$ connected with the transverse velocity created by the rotation, which undergoes an initial growth phase up to $\mathrm{Ta} \approx 3000$ and then a regular and moderate decrease. The initial decrease of $\mathrm{Gr}_{c 0} / \mathrm{Gr}_{c}$ is then due to the strong decrease of $R_{U}^{K}$ that overpasses the increase of $R_{V}^{K}$. In contrast, for large Ta, both terms contribute to the decrease of $\mathrm{Gr}_{c 0} / \mathrm{Gr}_{c}$, but $R_{V}^{K}$ is the unique contributor to the instability triggering. Note that the contribution related to the transverse basic velocity surpasses that related to the longitudinal basic velocity at $\mathrm{Ta} \approx 1800$.

We finally can deepen the analysis of the shear terms $K_{s, U}^{\prime \prime}$ and $K_{s, V}^{\prime \prime}$, which are the main parts of $R_{U}^{K}$ and $R_{V}^{K}$, respectively. The term $K_{s, U}^{\prime \prime}\left(K_{s, V}^{\prime \prime}\right)$ is given by the integral across the layer of $k_{s, U}^{\prime \prime}$ $\left(k_{s, V}^{\prime \prime}\right)$, which can itself be written as the product of two terms: $-\left(d u_{b} / d z\right)\left[-\left(d v_{b} / d z\right)\right]$, a quantity related to the analytical basic flow, which is independent of $\mathrm{Gr}_{c}$ and only dependent on Ta, and $\operatorname{Re}\left(w u^{*} /\left|K_{\mathrm{visc}}\right|\right)\left[\operatorname{Re}\left(w v^{*} /\left|K_{\mathrm{visc}}\right|\right)\right]$, a quantity related to the velocity disturbances at the threshold $\mathrm{Gr}_{c}$. These terms are given in Fig. 24. They are all symmetric with respect to $z=0$.

We consider the components related to $K_{s, U}^{\prime \prime}$ and given in Figs. 24(a) and 24(b). For Ta $=0$, we see that the main positive contribution to $K_{s, U}^{\prime \prime}$ comes from the positive values of $-d u_{b} / d z$ around the center associated with the maximum positive values of $\operatorname{Re}\left(w u^{*} /\left|K_{\mathrm{visc}}\right|\right)$, whereas the negative values of $-d u_{b} / d z$ along the walls are associated with rather small positive values of $\operatorname{Re}\left(w u^{*} /\left|K_{\mathrm{visc}}\right|\right)$ and will give only a small negative contribution. When the Taylor number is increased, $-d u_{b} / d z$ is drastically reduced at the center, even reaching values close to zero for Ta above 10000 . At the same time, the broad peak of $\operatorname{Re}\left(w u^{*} /\left|K_{\text {visc }}\right|\right)$ is first reduced in width, corresponding to a change of the perturbation structure, before decreasing in intensity. All these changes contribute together to the strong decrease of $K_{s, U}^{\prime \prime}$. Note that negative values of $\operatorname{Re}\left(w u^{*} /\left|K_{\text {visc }}\right|\right)$ are also observed at the half-distance between the center and the walls. Associated with positive values of $-d u_{b} / d z$, they will give the negative contribution to $K_{s, U}^{\prime \prime}$ observed in Fig. 22.

Concerning the components related to $K_{s, V}^{\prime \prime}$ and given in Figs. 24(c) and 24(d), they are indeed zero for $\mathrm{Ta}=0$ and will develop with the applied rotation, with a first quick phase of increase and a second phase of moderate decrease. The main positive contribution to $K_{s, V}^{\prime \prime}$ comes from 
(a)

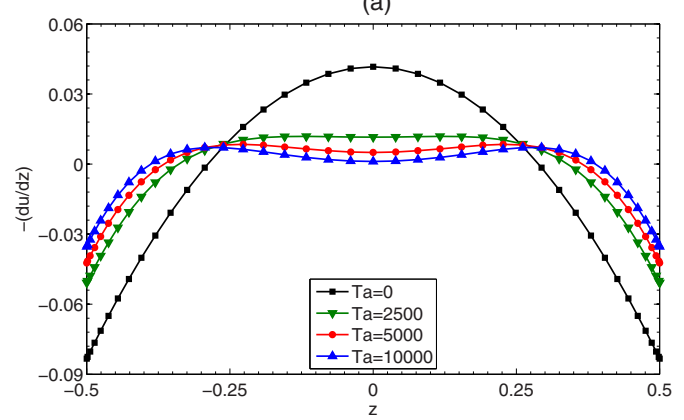

(c)

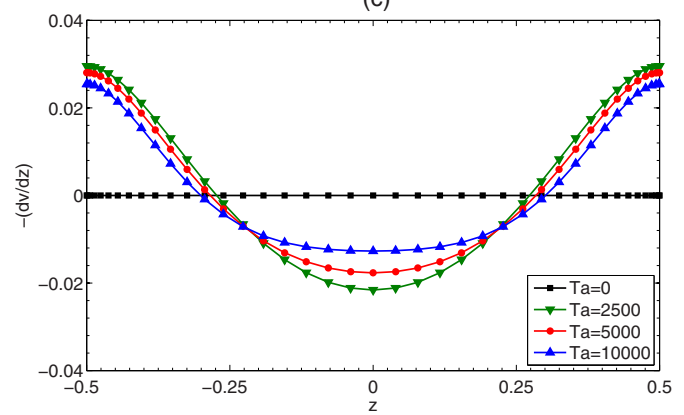

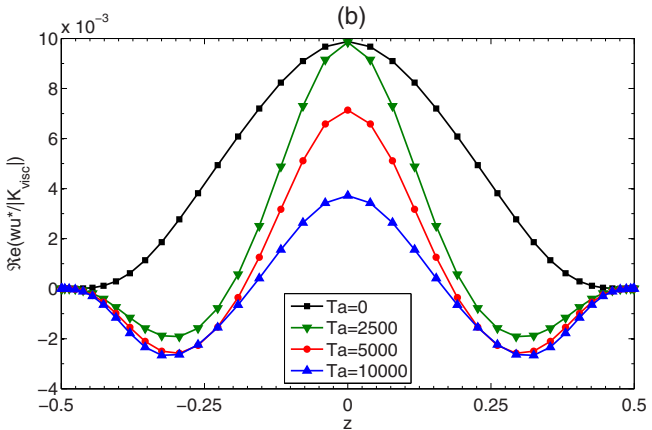

(d)

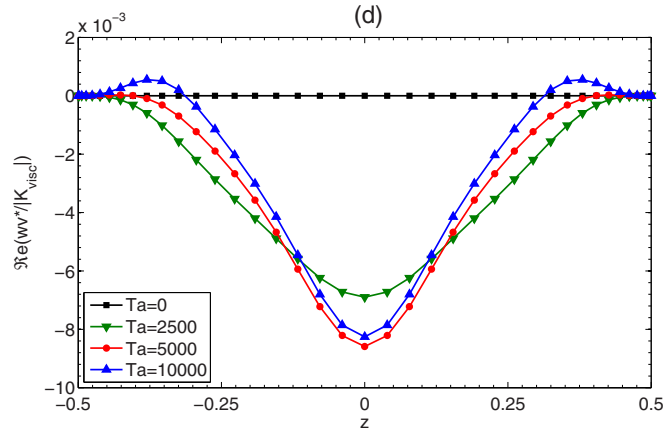

FIG. 24. Distributions along $z$ of (a) $-(d u / d z)$, (b) $\operatorname{Re}\left(w u^{*} /\left|E_{\mathrm{visc}}\right|\right),(\mathrm{c})-(d v / d z)$, and (d) $\operatorname{Re}\left(w v^{*} /\left|E_{\mathrm{visc}}\right|\right)$ for the shear instability at $\operatorname{Pr}=0.01$ and different values of Ta.

the negative values of $-d v_{b} / d z$ around the center associated with the maximum negative values of $\operatorname{Re}\left(w v^{*} /\left|K_{\text {visc }}\right|\right)$, whereas positive values of $-d v_{b} / d z$ along the walls are associated with rather small values of $\operatorname{Re}\left(w v^{*} /\left|K_{\text {visc }}\right|\right)$ and will give only a small negative contribution. When Ta is increased, the eventual decrease of these components remains moderate, explaining that $K_{s, V}^{\prime \prime}$ (i.e., the transverse velocity profile) keeps a destabilizing contribution at quite large Ta.

\section{B. Thermal (Rayleigh) instability}

For the thermal instability, contrarily to the shear instability, buoyancy is expected to play a role as this instability owes its existence to the unstable temperature stratification of the basic flow. We consider the total energy budgets for this Rayleigh instability as a function of the Prandtl number Pr ranging from 0.1 to 10 (Fig. 25). Two values of the Taylor number are considered: $\mathrm{Ta}=0$ (longitudinal rolls) and $\mathrm{Ta}=500$ (oblique rolls). In both cases, we observe a strong increase of the buoyancy term $K_{\text {buoy }}^{\prime}$ with the increase of the Prandtl number in the region $0.1<\operatorname{Pr}<3$, whereas the other terms (shear terms $K_{s, U}^{\prime}$ and $K_{s, V}^{\prime}$ ) are strongly damped. For $\operatorname{Pr}>3$, all the terms evolve more slowly and tend towards asymptotic values, 1 for $K_{\text {buoy }}^{\prime}$ and 0 for $K_{s, U}^{\prime}$ and $K_{s, V}^{\prime}$, indicating that buoyancy becomes the dominant term.

It can be interesting to see whether the thresholds for this Rayleigh instability can be obtained from a critical Rayleigh number $\mathrm{Ra}_{B}$ defined at the level of the stratified layers along the boundaries. Taking the case $\mathrm{Ta}=0$ as an example, we could write

$$
\operatorname{Ra}_{B}=\frac{g \gamma \Delta \tilde{T} L^{3}}{\kappa v}=1100,
$$

with the characteristic temperature difference $\Delta \tilde{T}=3.510^{-4} \operatorname{Gr} \operatorname{Pr} h|\vec{\nabla} \tilde{T}|_{0}$ (obtained from the dimensionless temperature gradient at the boundary taken over the unstably stratified height [Fig. 5(b)], multiplied by the reference temperature $h|\vec{\nabla} \tilde{T}|_{0}$ ) and $L=h / 4$. The value 1100 
EFFECT OF ROTATION ON THE STABILITY OF SIDE- . . .

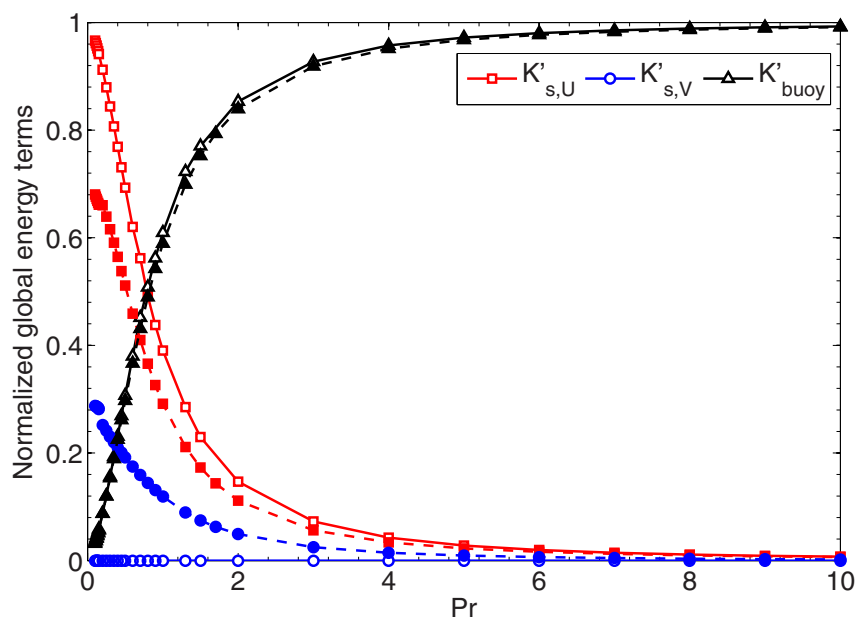

FIG. 25. Global energy terms $K_{i}^{\prime}$ versus the Prandtl number for Ta $=0$ (solid lines) and $\mathrm{Ta}=500$ (dashed lines).

corresponds to the critical Rayleigh number in an unstably stratified extended layer with rigid-free boundaries [22]. We then obtain

$$
\frac{3.5 \times 10^{-4}}{64} \operatorname{Gr} \operatorname{Pr} \frac{g \gamma|\vec{\nabla} \tilde{T}|_{0} h^{4}}{\kappa \nu}=1100,
$$

which finally gives

$$
\operatorname{Ra}_{c}=\mathrm{Gr}_{c} \operatorname{Pr}=800 \sqrt{\frac{1100}{3.5}} \approx 14180 .
$$

If we compare with the values of the critical Rayleigh number $\mathrm{Ra}_{c}$ for the Rayleigh instability expressed as a function of Pr in Fig. 26, we see that the value 14180 approximates quite well the

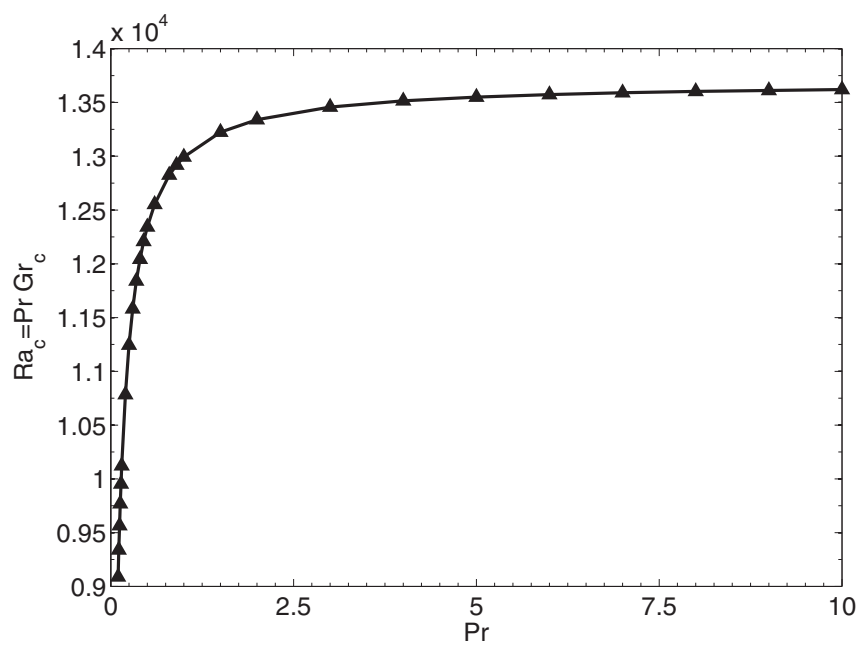

FIG. 26. Threshold for the Rayleigh instability expressed as the critical Rayleigh number $\mathrm{Ra}_{c}$ as a function of the Prandtl number $\operatorname{Pr}(\mathrm{Ta}=0)$. 
(a)

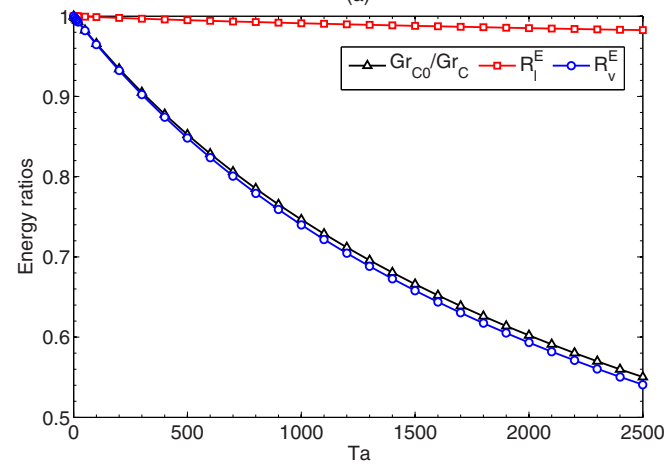

(b)

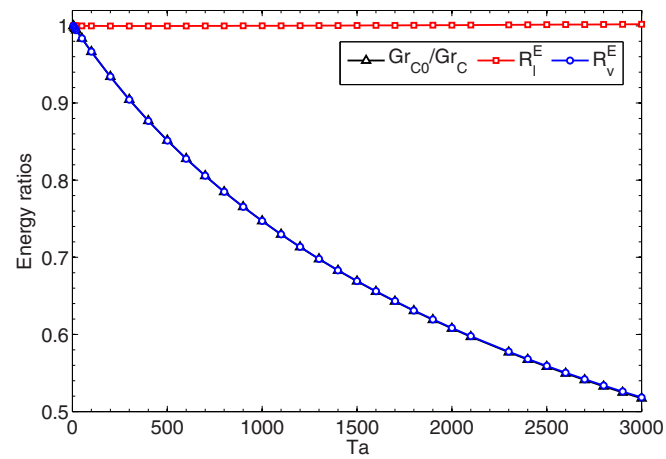

FIG. 27. Variation of the energy ratios $R_{l}^{E}$ and $R_{v}^{E}$ given by Eq. (33) as a function of the Taylor number Ta for the Rayleigh instability at threshold for (a) $\operatorname{Pr}=0.7$ and (b) $\operatorname{Pr}=7$.

values of $\mathrm{Ra}_{c}$ obtained asymptotically for large enough $\operatorname{Pr}(\operatorname{Pr}>3$, for example), in the domain where buoyancy is the main destabilizing force.

We can now analyze the influence of the rotation. In Fig. 27, we present the contributions to the critical Grashof number $R_{l}^{E}$ and $R_{v}^{E}$ obtained from the thermal energy analysis and defined in Eq. (33). For both values of $\operatorname{Pr}, R_{l}^{E}$ is almost constant and $R_{v}^{E}$ decreases as Ta is increased in a hyperbolic way.

The energy ratio $R_{v}^{E}$ is built on $E_{v}^{\prime \prime}$, which is the integral of a spatial field. This spatial field can be decomposed as a product of two terms, one related to the basic flow $\left(-d T_{b} / d z\right) / \mathrm{Gr} \operatorname{Pr}$ and the other related to the fluctuations at the critical threshold $\operatorname{Re}\left(\theta^{*} w\right) /\left|E_{d}\right|$. These two terms are presented in Fig. 28. We see that the decrease of $R_{v}^{E}$ observed when rotation is applied is due to the decrease of the basic temperature gradient $\left(-d T_{b} / d z\right) / \mathrm{Gr} \operatorname{Pr}$ attenuated by a slighter increase of the fluctuations product $\operatorname{Re}\left(\theta^{*} w\right) /\left|E_{d}\right|$. For both values of $\operatorname{Pr}$, the same type of change with Ta is observed.

\section{Oscillatory instability}

The global kinetic energy contributions for the oscillatory instability are given in Fig. 29(a) as a function of the Taylor number for both the adiabatic case at $\operatorname{Pr}=0.05$ and the conducting case at $\operatorname{Pr}=0.15$. We see that for this oscillatory instability, both shear and buoyancy contributions are involved. The longitudinal shear term $K_{s, U}^{\prime}$ will decrease with the applied rotation, whereas the
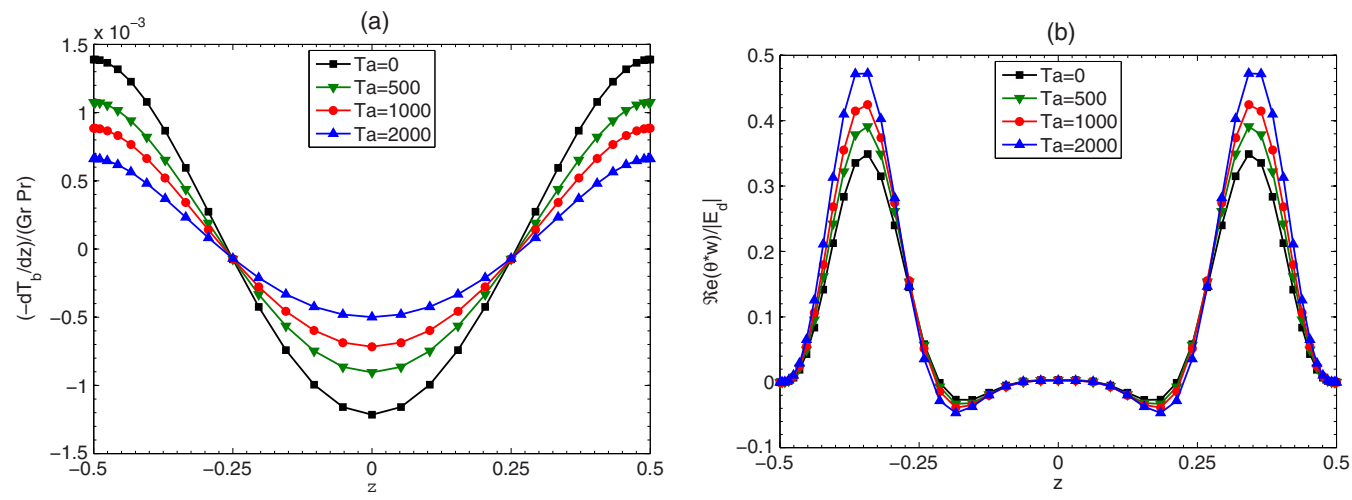

FIG. 28. For the Rayleigh instability at threshold $(\operatorname{Pr}=0.7)$, $z$ profiles of (a) the basic temperature gradient $\left(-d T_{b} / d z\right) / \operatorname{Gr} \operatorname{Pr}$ and (b) the fluctuation product $\operatorname{Re}\left(\theta^{*} w\right) /\left|E_{d}\right|$, which by integration on the fluid layer give $E_{v}^{\prime \prime}$. 

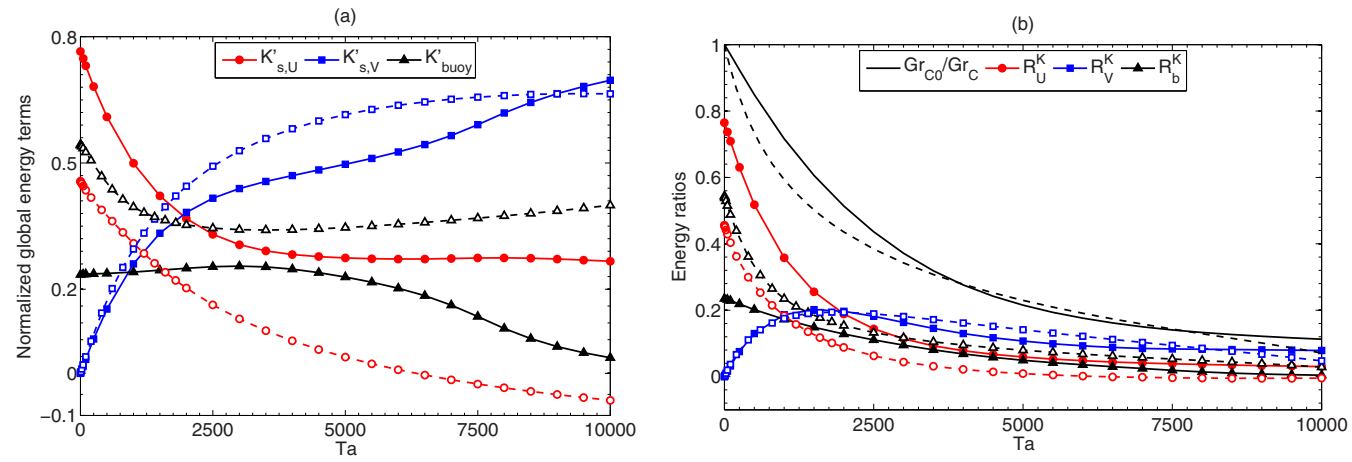

FIG. 29. (a) Contributions to the total fluctuating kinetic energy and (b) ratios $R_{U}^{K}, R_{V}^{K}$, and $R_{b}^{K}$ defining $\mathrm{Gr}_{c 0} / \mathrm{Gr}_{c}$ as a function of the Taylor number for the oscillatory instability in the adiabatic case $(\operatorname{Pr}=0.05$, dashed lines) and in the conducting case ( $\operatorname{Pr}=0.15$, solid lines).

transverse shear term $K_{s, V}^{\prime}$ will increase from zero and eventually become dominant. The buoyancy term evolves differently depending on the cases considered. For the conducting case at $\operatorname{Pr}=0.15$, the buoyancy contribution is smaller than $K_{s, U}^{\prime}$ without rotation and remains smaller when rotation is applied. It is initially almost constant up to $\mathrm{Ta}=5000$ and then decreases to give a small contribution at $\mathrm{Ta}=10000$. In contrast, for the adiabatic case at $\mathrm{Pr}=0.05$, the buoyancy contribution remains larger than $K_{s, U}^{\prime}$, which even decreases towards negative values (small stabilizing contribution). This buoyancy contribution first decreases with the increase of Ta before slightly increasing, thus remaining a non-negligible contribution.

To better show how these energy terms contribute to the observed increase of the threshold $\mathrm{Gr}_{c}$ with Ta, Fig. 29(b) gives the ratios $R_{U}^{K}, R_{V}^{K}$, and $R_{b}^{K}$ defined in Eq. (32), as well as $\mathrm{Gr}_{c 0} / \mathrm{Gr}_{c}$, as a function of the Taylor number, for the two previous cases. In both cases, the stabilization effect obtained when Ta is increased (decrease of the quantity $\mathrm{Gr}_{c 0} / \mathrm{Gr}_{c}$ ) is due to the strong decrease of $R_{U}^{K}$ and $R_{b}^{K}$, attenuated by the initial increase of $R_{V}^{K}$.

\section{CONCLUSION}

Rotation occurs in many geophysical situations and in engineering applications where flows initiated by temperature gradients are present. It is known that this rotation can affect the flow dynamics through the action of the noninertial Coriolis forces and it is this effect we wanted to study here. Our paper focused on a simple heated situation, more precisely, the buoyant convection generated by a horizontal gradient of temperature in an infinite fluid layer, which is known as the Hadley circulation, and studied the effects induced by applying a rotation around the vertical axis.

First, the basic flow profile with rotation was derived and the influence of the rotation, quantified by the Taylor number Ta, was depicted. The original longitudinal velocity profile is decreased in intensity when rotation is applied and its structure is progressively changed, with eventually a no-flow central zone and boundary layers along the top and bottom walls. In addition, a transverse velocity component is created, which first increases with the rotation intensity, overcomes the longitudinal velocity, and then decreases. Different asymptotic behaviors for these velocity components were revealed: The initial increase of the transverse component occurs as $\mathrm{Ta}^{1 / 2}$ and the departure of the longitudinal component from the Hadley profile as Ta, whereas the asymptotic decrease at large Ta for these two components occurs as $\mathrm{Ta}^{-1 / 2}$.

The stability of these flows was then studied. The three different instabilities obtained without rotation for different Prandtl numbers (steady shear instability, oscillatory instability, and steady Rayleigh instability) are all stabilized by the rotation. The stabilization is connected with the decrease of the longitudinal velocity component of the basic flow when rotation is applied. For 


\section{A. MEDELFEF et al.}

the shear instability, the main contribution to the instability is the energy based on the shear of the basic flow, which decreases as the rotation is increased and changes from being dominated by the longitudinal component to being dominated by the transverse component created by the rotation. For the oscillatory instability, we observe the same change of influence between the longitudinal and transverse velocity components, but a destabilizing buoyancy contribution is also involved. Finally, for the Rayleigh instability, the stabilization is related to the decrease of the destabilizing thermal energy term connected with the vertical basic temperature gradient. In all cases, the increase of the critical thresholds is accompanied by a spinning of the wave vector (originally oriented along the main axes) corresponding to a progressive change of the orientation of the marginal perturbation rolls due to the applied rotation. The spinning is particularly important for the shear instabilities, initially corresponding to transverse rolls.

The next step for this study on the influence of rotation on thermal convection would be to consider three-dimensional cavities differentially heated between opposite vertical walls.

\section{ACKNOWLEDGMENT}

This collaborative work was supported by the PHC Maghreb Partnership Program No. 36951NG.

[1] G. S. Hadley, Concerning the cause of the general trade winds, Philos. Trans. 29, 58 (1735).

[2] D. Fultz, Experimental analogies to atmospheric motions, in Compendium of Meteorology, edited by T. F. Malone (American Meteorological Society, Boston, 1951), pp. 1235-1248.

[3] M. Ghil, P. Read, and L. Smith, Geophysical flows as dynamical systems: The influence of Hide's experiments, Astron. Geophys. 51, 4.28 (2010).

[4] R. Hide, Path of discovery in geophysical fluid dynamics, Astron. Geophys. 51, 4.16 (2010).

[5] G. Dhanaraj, K. Byrappa, V. Prasad, and M. Dudley, Springer Handbook of Crystal Growth (Springer, Berlin, 2010).

[6] D. T. J. Hurle, Temperature oscillations in molten metals and their relationship to the growth state in melt grown crystals, Philos. Mag. 13, 305 (1966).

[7] A. E. Gill, A theory of thermal oscillations in liquid metals, J. Fluid Mech. 64, 577 (1974).

[8] J. E. Hart, Stability of thin non-rotating Hadley circulations, J. Atmos. Sci. 29, 687 (1972).

[9] P. Laure and B. Roux, Linear and non-linear analysis of the Hadley circulation, J. Cryst. Growth 97, 226 (1989).

[10] P. Laure, Étude des mouvements de convection dans une cavité rectangulaire soumise à un gradient de température horizontal, J. Theor. Appl. Mech. 6, 351 (1987).

[11] G. Gershuni, P. Laure, V. Myznikov, B. Roux, and E. Zhukhovitsky, On the stability of plane-parallel advective flows in long horizontal layers, Microgravity Q. 2, 141 (1992).

[12] S. M. Pimputkar and S. Ostrach, Convective effects in crystals grown from melts, J. Cryst. Growth 55, 614 (1981).

[13] M. Lappa, Secondary and oscillatory gravitational instabilities in canonical three-dimensional models of crystal growth from the melt. Part 2: Lateral heating and Hadley circulation, C. R. Mec., 335, 261 (2007).

[14] S. Kaddeche, D. Henry, and H. Ben Hadid, Magnetic stabilization of the buoyant convection between infinite horizontal walls with a horizontal temperature gradient, J. Fluid Mech. 480, 185 (2003).

[15] D. Henry, S. Kaddeche, and H. Ben Hadid, Stabilization of thermogravitational flows by magnetic field and surface tension, Phys. Fluids 17, 054106 (2005).

[16] A. Hudoba, S. Molokov, S. Aleksandrova, and A. Pedcenko, Linear stability of buoyant convection in a horizontal layer of an electrically conducting fluid in moderate and high vertical magnetic field, Phys. Fluids 28, 094104 (2016). 


\section{EFFECT OF ROTATION ON THE STABILITY OF SIDE- . . .}

[17] W. Dridi, D. Henry, and H. Ben, Hadid Influence of acoustic streaming on the stability of melt flows in horizontal Bridgman configurations, J. Cryst. Growth 310, 1546 (2008).

[18] W. Dridi, D. Henry, and H. Ben Hadid, Stability of buoyant convection in a layer submitted to acoustic streaming, Phys. Rev. E 81, 056309 (2010).

[19] P. H. Stone, Some properties of Hadley regimes on rotating and non-rotating planets, J. Atmos. Sci. 25, 644 (1968).

[20] P. H. Stone, The structure and circulation of the deep Venus atmosphere, J. Atmos. Sci. 31, 1681 (1974).

[21] Y. Nakagawa and P. Frenzen, A theoretical and experimental study of cellular convection in rotating fluids, Q. J. Geophys. 7, 1 (1955).

[22] S. Chandrasekhar, Hydrodynamic and Hydromagnetic Stability (Oxford University Press, Oxford, 1961).

[23] K. Zhang and P. H. Robert, Thermal inertial waves in a rotating fluid layer: Exact and asymptotic solutions, Phys. Fluids 9, 1980 (1997).

[24] S. N. Aristov and P. G. Frik, Large-scale turbulence in a thin layer of nonisothermal rotating fluid, Fluid Dyn. 23, 522 (1988).

[25] K. G. Schwarz, Effect of rotation on the stability of advective flow in a horizontal fluid layer at a small Prandtl number, Fluid Dyn. 40, 193 (2005).

[26] K. G. Shvarts and A. Boudlal, Effect of rotation on stability of advective flow in horizontal liquid layer with a free upper boundary, J. Phys.: Conf. Ser. 216, 012005 (2010).

[27] K. G. Shvarts, Influence of slow rotation on the stability of a thermocapillary incompressible liquid flow in an infinite layer under zero-gravity conditions for small Prandtl number, Fluid Dyn. Res. 44, 031416 (2012).

[28] D. G. Chikulaev and K. G. Shvarts, Effect of rotation on the stability of advective flow in a horizontal liquid layer with solid boundaries at small Prandtl numbers, Fluid Dyn. 50, 215 (2015).

[29] N. S. Knutova and K. G. Shvarts, A study of behavior and stability of an advective thermocapillary flow in a weakly rotating liquid layer under microgravity, Fluid Dyn. 50, 340 (2015).

[30] A. Mehdizadeh and M. Oberlack, Analytical and numerical investigations of laminar and turbulent Poiseuille-Ekman flow at different rotation rates, Phys. Fluids 22, 105104 (2010).

[31] J. C. Lagarias, J. A. Reeds, M. H. Wright, and P. E. Wright, Convergence properties of the Nelder-Mead simplex method in low dimensions, SIAM J. Optimiz. 9, 1.112 (1998).

[32] J. H. Mathews and K. D. Fink, Numerical Methods Using Matlab (Prentice Hall, Englewood Cliffs, 1999). 